\title{
RELATIVISTIC CONTINUUM IN SPECIFIED FORCE FIELD
}

\section{Stanislav A. Podosenov, Elena R. Men'kova}

All-Russian Research Institute for Optical and Physical Measurements, http://www.vniiofi.ru

Moscow 119361, Russian Federation

\section{Alexander A. Potapov \\ Kotel'nikov Institute of Radioengineering and Electronics, Russian Academy of Sciences, http://www.cplire.ru Moscow 125009, Russian Federation \\ Jinan University, College of Information Science and Technology/College of Cyber Security, http://www. jnu.edu.cn Guangzhou 510632, China \\ Cooperative Chinese-Russian Laboratory of Informational Technologies and Signals Fractal Processing, Guangzhou 510632, China}

\section{Jaykov Foukzon}

Center for Mathematical Sciences, Israel Institute of Technology, http://www.technion.ac.il

Haifa 3200003, Israel

podosenov@mail.ru,potapov@cplire.ru,jaykovfoukzon@list.ru,e_menkova@mail.ru

Abstract. A continuum motion in the flat Minkowski space in the specified force field is considered. When movement the relative space-time curvature stipulated by the noncoincidence of the hypersurface orthogonal to world lines of basis particles with the simultaneity hypersurface arises. The anholonomic mathematical apparatus and its continua application is developed. Metrics for relative 4-interval element squares for the arbitrary continuum motion and special cases are found. The acceleration addition law is obtained. It turns out that exact solutions of the Einstein's equations for the isotropic cosmological model for the strongly evacuated gas and for the critical density follow from solutions of the Newtonian gas dynamics Euler's equations.

Keywords: space-time, curvature tensor, reference frame, Born's rigidity, Minkowski, Riemann, Einstein, coordinates, Christoffel, hypersurfaces.

UDC 530.12, 531.134, 537.9

Bibliography - 25 references

Received 10.11.2017

RENSIT, 2017, 9(2):161-180

DOI: $10.17725 /$ rensit.2017.09.161

\section{CONTENTS}

1. INTRODUCTION (161)

2. Relative NRF CURVATURe tensor IN SPECIAL RELATIVITY (SR) (162)

3. Element square of RELATIVE CONTINUUM INTERVAL IN LAGRANGIAN COORDINATES (170)

3.1. Uniformly rotating RF (170)

3.2. Relativistic (nonrigid) uniformly accelerated NRF (171)

4. Acceleration addition law, Relative NRF CURVATURE TENSOR IN MINKOWSKI SPACE (172)

5. RElative CONTINUUM CURVATURE TENSOR IN Newton MECHanics (175)

6. Conclusion (178)

REFERENCES (178)

\section{INTRODUCTION}

Generally a continuum motion description in an inertial reference frame (IRF) and a transition to a noninertial reference frame (NRF) demands a going out of the flat space-time [1]. That is connected with both a specifying of a force field affecting on the medium particles and a condition superposition on motion continuum characteristic by means of the structure equations [1-5]. These equations connect the Riemannian-Christoffel tensor and the strain velocity tensors, the rotational velocity tensors and the first curvature vectors of medium particle world lines. As a result the set of equations is an overdetermined system and it is not realized in Minkowski space. 
This system is solved when considering of a medium motion in the Riemannian space or in a general case in the metric connectivity space. However if one does not impose characteristic continuum additional conditions and restrict just an integration of motion equations, for example, in a flat space-time, then no going out of the flat space-time is occur. When using nonholonomic transformations the curvature tensor obtained from Minkowski space in the nonholonomic coordinates is identically zero. However this zero tensor can be divided into two nonzero parts. One of them is expressed in an ordinary way with the Christoffel symbols and the other one depends on the moving medium characteristics [2-5].

\section{RELATIVE NRF CURVATURE}

\section{TENSOR IN SPECIAL RELATIVITY} (SR)

The essence of our NRF mapping method from IRF is in finding of transformation rules of geometric objects prescribed in Galilean coordinates of Minkowski space (Euler variables) by means of affine frames of Lagrangian comoving NRF. First this method was developed in [6]. "Space" reference marks of the NRF are located in hypersurfaces orthogonal to world lines of medium particles (anholonomic in the presence of rotations) and time vectors coincide with the field of 4 velocities $V^{\mu}$ tangent to world lines.

The continuum motion law in an arbitrary force field in Minkowski space is described by equations

$$
x^{\mu}=\Psi^{\mu}\left(y^{\hat{k}}, \xi^{\hat{0}}\right),
$$

where $x^{\mu}$ are the Eulerian coordinates and $y^{\hat{k}}$ are the Lagrangian coordinates which are constant along each fixed world line, $(1 / \mathrm{c}) \xi^{\hat{0}}$ is the time parameter, for example, proper time. Let $\mu$ indices belong to Eulerian coordinates and $\hat{\mu}$ indices belong to Lagrangian ones. Differentiating (1) with respect to $y^{\hat{k}}$ and $\xi^{\hat{0}}$ in each space-time point, we obtain the affine frame. We note that in the general case time $\partial x^{\mu} / \partial \xi^{\hat{0}}$ and space $\partial x^{\mu} / \partial y^{\hat{k}}$ vectors are not orthogonal to each other. However, the reference marks with orthogonal "time" and "space" vectors can be constructed from relation (1), but these reference marks are not a result of 4-radius vector differentiation with respect to $y^{\hat{k}}$ and $\xi^{\hat{0}}$ Lagrangian coordinates. These reference marks are anholonomic and corresponding Lame coefficients have the form

$$
\begin{aligned}
& h_{\hat{k}}^{\mu}=\left(\delta_{\varepsilon}^{\mu}-V^{\mu} V_{\varepsilon}\right) \frac{\partial \Psi^{\varepsilon}}{\partial y^{\hat{k}}}, h_{\hat{0}}^{\mu}=\frac{\partial \Psi^{\mu}}{\partial \xi^{\hat{0}}}=V^{\mu}, \\
& h_{\mu}^{\hat{k}}=\frac{\partial y^{\hat{k}}}{\partial x^{\mu}}, h_{\mu}^{\hat{0}}=V_{\mu} .
\end{aligned}
$$

For anholonomic coordinates connectivity coefficients $\Gamma_{\hat{\alpha} \hat{b}}^{\hat{\sigma}}$ in Minkowski space can be presented in the form [7]

$$
\Gamma_{\hat{\alpha} \hat{b}}^{\hat{\sigma}}=h_{\varepsilon}^{\hat{\sigma}} \frac{\hat{\partial} h_{\hat{b}}^{\varepsilon}}{\hat{\partial} y^{\hat{\alpha}}}=-h_{\hat{b}}^{\varepsilon} \frac{\hat{\partial} h_{\varepsilon}^{\hat{\sigma}}}{\hat{\partial} y^{\hat{\alpha}}},
$$

where hereinafter $\hat{\partial} / \hat{\partial} y^{\hat{\alpha}}$ will be directional derivatives determined as

$$
\frac{\hat{\partial}}{\partial y^{\hat{0}}}=\frac{\hat{\partial}}{\hat{\partial} \xi^{\hat{0}}}=V^{\mu} \frac{\partial}{\partial x^{\mu}}, \frac{\hat{\partial}}{\hat{\partial} y^{\hat{k}}}=h_{\hat{k}}^{\mu} \frac{\partial}{\partial x^{\mu}} \text {. }
$$

It is proposed in formula (3) that Galilean coordinates have been selected in Minkowski space. For arbitrary curvilinear coordinates the connectivity formula has the form

$$
\Gamma_{\hat{\alpha} \hat{b}}^{\hat{\sigma}}=-h_{\hat{b}}^{\varepsilon} \frac{\hat{\partial} h_{\varepsilon}^{\hat{\sigma}}}{\hat{\partial} y^{\hat{\alpha}}}+\Gamma_{\nu \lambda}^{\mu} h_{\mu}^{\hat{\sigma}} h_{\hat{b}}^{v} h_{\hat{\alpha}}^{\lambda},
$$

where $\Gamma_{v \lambda}^{\mu}$ is the connectivity in Minkowski space. We form the nonholonomity object $C_{\hat{\alpha} \hat{b}}^{\hat{\sigma}}$ from Lame coefficients

$$
\begin{aligned}
& C_{\hat{\alpha} \hat{b} .}^{\hat{\sigma}}=-\Gamma_{[\hat{\alpha} \hat{b}]}^{\hat{\sigma}}=\frac{1}{2}\left(h_{\hat{b}}^{\varepsilon} \frac{\hat{\partial} h_{\varepsilon}^{\hat{\sigma}}}{\partial y^{\hat{\alpha}}}-h_{\hat{\alpha}}^{\varepsilon} \frac{\hat{\partial} h_{\varepsilon}^{\hat{\sigma}}}{\hat{\partial} y^{\hat{b}}}\right)= \\
& =\frac{1}{2} h_{\hat{\alpha}}^{v} h_{\hat{b}}^{\varepsilon}\left(\frac{\partial h_{\varepsilon}^{\hat{\sigma}}}{\partial x^{\nu}}-\frac{\partial h_{v}^{\hat{\sigma}}}{\partial x^{\varepsilon}}\right) .
\end{aligned}
$$


Following to Schouten [7] for anholonomic transformations the $\Gamma_{\hat{\alpha} \hat{b}}^{\hat{\sigma}}$ connectivity has the form

$$
\begin{aligned}
& \Gamma_{\hat{\alpha} \hat{b}}^{\hat{\sigma}}=\left\{\begin{array}{c}
\hat{\sigma} \\
\hat{\alpha} \hat{b}
\end{array}\right\}+\mathrm{T}_{\hat{\alpha} \hat{b}}^{\hat{\sigma}}, \mathrm{T}_{\hat{\alpha} \hat{b}}^{\hat{\sigma}}=-C_{\hat{\alpha} \hat{b}}^{\hat{\sigma}}+ \\
& +g_{\hat{\alpha} \hat{\varepsilon}}{ }^{\hat{\hat{\sigma}} \hat{\sigma}} C_{\hat{b} \hat{v}}^{\hat{\varepsilon}}+g_{\hat{b} \hat{\varepsilon}} g^{\hat{\sigma} \hat{v}} C_{\hat{\alpha} \hat{v}}^{\hat{\hat{v}}} .
\end{aligned}
$$

If one calculates the Riemannian-Christoffel's tensor in Minkowski space, it is identically zero. It is clear that the transition into the Lagrangian comoving NRF by means of Lame coefficients (2) does not make the curvature tensor differed from zero and it results in identity [7]

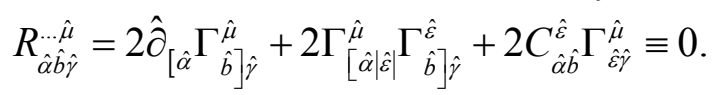

It follows from (6) and (7) that

$$
\hat{R}_{\hat{\alpha} \hat{b} \hat{\gamma}}^{\cdots, \hat{\mu}}=-2 \hat{\nabla}_{[\hat{\alpha}} \mathrm{T}_{\hat{b}] \hat{\gamma}}^{\hat{\mu}}-2 \mathrm{~T}_{[\hat{\alpha}|\hat{\alpha}| \hat{b}] \hat{\hat{b}}] \hat{\gamma}}^{\hat{\hat{\gamma}}}-2 C_{\hat{\alpha} \hat{b}}^{\hat{\hat{b}}} \mathrm{~T}_{\hat{\alpha} \gamma}^{\hat{\mu}} .
$$

In relation (8) the curvature tensor is calculated by means of Christoffel's symbols $\left\{\begin{array}{c}\hat{\sigma} \\ \hat{\alpha} \hat{b}\end{array}\right\}$, obtained from metric coefficients

$$
\hat{g}_{\hat{\alpha} \hat{b}}=g_{\mu \nu} h_{\hat{\alpha}}^{\mu} h_{\hat{b}}^{v}, \hat{g} 0_{\hat{0} \hat{0}}=1, \hat{g}_{\hat{0} \hat{k}}=0 \text {, }
$$

where $g_{\mu \nu}$ is the metric tensor in Eulerian coordinates of Minkowski space. Christoffel's symbols are calculated in the usual way with the substitution of partial derivatives by directional derivatives, and $\hat{\nabla}_{\hat{\alpha}}$ operator is calculated by means of the Christoffel's connectivity.

Thus, anholonomic transformations resulted in the curvature tensor differed from zero and calculated by means of the Christoffel's connectivity (6).

As it would be followed further from the analysis of motion equations, $\hat{R}_{\hat{\alpha} \hat{b} \hat{y}}^{. \hat{\mu}}$ curvature tensor may be called as relative NRF curvature tensor.

For anholonomic coordinates following commutation relations exist [7]

$$
\frac{\hat{\partial}^{2}}{\hat{\partial} y^{\hat{b}} \hat{\partial} y^{\hat{\alpha}}}-\frac{\hat{\partial}^{2}}{\hat{\partial} y^{\hat{\alpha}} \hat{\partial} y^{\hat{b}}}=2 C_{\hat{\alpha} \hat{b}}^{\hat{\partial}} \frac{\hat{\partial}}{\hat{\partial} y^{\hat{\gamma}}} \text {. }
$$

The concrete form of a nonholonomity object depends on selected Lame coefficients which are determined against the time parameter choice along world lines of basis particles. For example, if one selects a proper time as a time parameter (2) then the calculation of the nonholonomity object results in relations

$$
C_{\hat{k} \hat{l}}^{\hat{0}}=\Omega_{\hat{k} \hat{l}}, 2 C_{\hat{0} \hat{k}}^{\hat{0}}=F_{\hat{k}}, C_{\hat{\alpha} \hat{b}}^{\hat{k}}=0 \text {, }
$$

where

$$
\Omega_{\hat{k} \hat{l}}=\Omega_{\mu \nu} h_{\hat{k}}^{\mu} h_{\hat{l}}^{v}, F_{\hat{k}}=F_{\mu} h_{\hat{k}}^{\mu} .
$$

In receiving (12) one can use relations, where the rotational velocity tensor and the vector of 4-acceleration are considered in Eulerian coordinates of Minkowski space and they are projected with Lame parameters into the comoving Lagrangian NRF. For the nonholonomity object (11) commutation relations (10) reduce to the form

$$
\begin{aligned}
& \frac{\hat{\partial}^{2}}{\hat{\partial} y^{\hat{k}} \hat{\partial} y^{\hat{l}}}-\frac{\hat{\partial}^{2}}{\hat{\partial} y^{\hat{l}} \hat{\partial} y^{\hat{k}}}=2 \Omega_{\hat{l} \hat{k}} \frac{\hat{\partial}}{\partial y^{\hat{0}}}, \\
& \frac{\hat{\partial}^{2}}{\hat{\partial} y^{\hat{k}} \hat{\partial} y^{\hat{0}}}-\frac{\hat{\partial}^{2}}{\hat{\partial} y^{\hat{0}} \hat{\partial} y^{\hat{k}}}=F_{\hat{k}} \frac{\hat{\partial}}{\hat{\partial} y^{\hat{0}}} .
\end{aligned}
$$

Commutation relations (13) are equivalent to Zel'manov commutation relations [8]. From metric (9), expansion (6) and Lame coefficients (2) we obtain

$$
\begin{aligned}
& \left\{\begin{array}{c}
\hat{0} \\
\hat{o} \hat{o}
\end{array}\right\}=\left\{\begin{array}{c}
\hat{k} \\
\hat{o} \hat{o}
\end{array}\right\}=\left\{\begin{array}{c}
\hat{0} \\
\hat{o} \hat{k}
\end{array}\right\}=0,\left\{\begin{array}{c}
\hat{o} \\
\hat{k} \hat{k} \hat{l}
\end{array}\right\}=-\Sigma_{\hat{\hat{k}} \hat{l}}, \\
& \left\{\begin{array}{c}
\hat{k} \\
\hat{n} \hat{\imath}
\end{array}\right\}=\lambda_{\hat{n} \hat{i}}^{\hat{k}},\left\{\begin{array}{c}
\hat{k} \\
\hat{0} \hat{n}\}
\end{array}\right\}=\sum_{\hat{n}}^{\hat{k}}, \mathrm{~T}_{\hat{0} \hat{k} .}^{\hat{0}}=-F_{\hat{k}}, \mathrm{~T}_{\hat{0} \hat{\hat{o}} .}^{\hat{k}}=F^{\hat{k}}, \\
& \mathrm{~T}_{\hat{m} \hat{l} .}^{\hat{k}}=\mathrm{T}_{\hat{m} \hat{l} \hat{k}}=\mathrm{T}_{\hat{k} \hat{0} .}^{\hat{0}}=0, \mathrm{~T}_{\hat{0} \hat{l}}^{\hat{k}}=\mathrm{T}_{\hat{i} \hat{k}}^{\hat{k}}=\Omega_{\hat{l}}^{\hat{k}}, \mathrm{~T}_{\hat{k} \hat{l}}^{\hat{0}}=-\Omega_{\hat{\hat{l}} \hat{l}} \text {. }
\end{aligned}
$$

As

$$
\Sigma_{\hat{k} \hat{l}}+\Omega_{\hat{k} \hat{l}}=h_{\hat{k}}^{v} h_{\hat{l}}^{\mu} \nabla_{\mu} V_{v},
$$

one could show differentiating with respect to $y^{\hat{0}}$ that the following kinematic identity exists

$$
\begin{aligned}
& \frac{\hat{\partial}}{\hat{\partial} y^{\hat{0}}}\left(\Sigma_{\hat{k} \hat{l}}+\Omega_{\hat{k} \hat{l}}\right) \equiv \\
& \equiv \hat{g}^{\hat{m} \hat{n}}\left(\Sigma_{\hat{\mathfrak{l}} \hat{n}}+\Omega_{\hat{\hat{n}} \hat{n}}\right)\left(\Sigma_{\hat{k} \hat{m}}+\Omega_{\hat{k} \hat{m}}\right)+\hat{\nabla}_{\hat{k}} F_{\hat{l}}-F_{\hat{k}} F_{\hat{l}},
\end{aligned}
$$

whence alternating we obtain

$$
\frac{\hat{\partial}}{\hat{\partial} y^{\hat{0}}} \Omega_{\hat{k} \hat{l}} \equiv \hat{\nabla}_{[\hat{k}} F_{\hat{\imath}]} \text {. }
$$

Balancing of expression (16) gives 


$$
\begin{aligned}
& \frac{\hat{\partial}}{\hat{\partial} y^{\hat{0}}} \Sigma_{\hat{k} \hat{l}} \equiv \\
& \equiv \hat{g}^{\hat{m} \hat{n}}\left(\Sigma_{\hat{\hat{n}} \hat{n}}+\Omega_{\hat{\hat{l}} \hat{n}}\right)\left(\Sigma_{\hat{k} \hat{m}}+\Omega_{\hat{k} \hat{m}}\right)+\hat{\nabla}_{(\hat{k}} F_{\hat{l})}-F_{\hat{k}} F_{\hat{l}} .
\end{aligned}
$$

Although the relative NRF curvature tensor is calculated by means of Christoffel's symbols in the same way as an usual curvature tensor in the Riemannian space, however in expressing of a connectivity by a metric tensor, directional derivatives are used instead of usual partial derivatives. Therefore, the relative curvature tensor has features, which arise because of noncommutativity of directional derivatives. For example, the known Richi identity will have the form

$$
\begin{aligned}
& \hat{R}_{\hat{\alpha} \hat{b}, \hat{\gamma}}^{\cdots \hat{\mu}}+\hat{R}_{\hat{b} \hat{\gamma}, \hat{\alpha}}^{\cdots \hat{\alpha}}+\hat{R}_{\hat{\gamma} \hat{\alpha}, \hat{b}}^{\cdots \hat{b}}=
\end{aligned}
$$

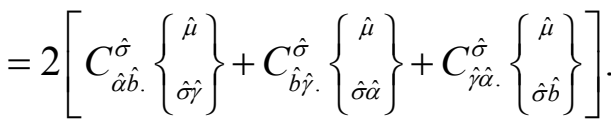

For Bianchi identity we have the expression $\hat{\nabla}_{\hat{\varepsilon}} \hat{R}_{\hat{\alpha} \hat{b}, \hat{\gamma}}^{\ldots \hat{\mu}}+\hat{\nabla}_{\hat{\alpha}} \hat{R}_{\hat{b} \hat{\varepsilon}, \hat{\gamma}}^{\ldots \hat{\mu}}+\hat{\nabla}_{\hat{b}} \hat{R}_{\hat{\varepsilon} \hat{\alpha}, \hat{\gamma}}^{\ldots \hat{\mu}}=$ $=2 C_{\hat{\varepsilon} \hat{\alpha} .}^{\hat{\sigma}} \hat{R}_{\hat{b} \hat{\sigma}, \hat{\gamma}}^{\ldots \hat{\mu}}+2 C_{\hat{\alpha} \hat{b} .}^{\hat{\sigma}} \hat{R}_{\hat{\varepsilon} \hat{\sigma}, \hat{\gamma}}^{\ldots \hat{\mu}}+2 C_{\hat{b} \hat{\varepsilon} .}^{\hat{\sigma} \hat{\varepsilon}} \hat{R}_{\hat{\alpha} \hat{\sigma}, \hat{\gamma}}^{\ldots \hat{\mu}}$,

to prove that it is convenient to pass into the local geodesic coordinate system.

One can represent the curvature tensor (8) in other equivalent form

$$
\begin{aligned}
& \hat{R}_{\hat{\alpha} \hat{b}, \hat{\gamma}}^{\cdots \hat{\mu}}=2 \hat{\partial}_{[\hat{\alpha}}\left\{\begin{array}{c}
\hat{\mu} \\
\hat{b}] \hat{\gamma}
\end{array}\right\}+2\left\{\begin{array}{c}
\hat{\mu} \\
{[\hat{\alpha}|\varepsilon|}
\end{array}\right\}\left\{\begin{array}{c}
\hat{\varepsilon} \\
\hat{b}] \hat{\gamma}
\end{array}\right\}+2 C_{\hat{\alpha} \hat{b} .}^{\hat{b} .}\left\{\begin{array}{c}
\hat{\mu} \\
\hat{\varepsilon} \hat{\gamma}
\end{array}\right\} \equiv \\
& \left.\equiv K_{\hat{\alpha} \hat{b}, \hat{\gamma}}^{\cdots \hat{\mu}}+2 C_{\hat{\alpha} \hat{b} .}^{\hat{b} .} \begin{array}{c}
\hat{\mu} \\
\hat{\varepsilon} \hat{\gamma}
\end{array}\right\} . \\
& K_{\hat{\alpha} \hat{b}, \hat{\gamma}}^{\cdots \hat{\mu}} \text { is not a tensor relatively holonomic }
\end{aligned}
$$
transformations of Lagrangian variables, although its form is not differed from an usual curvature tensor. However substitution of partial derivatives by directional derivatives results in the modification of transformation properties.

As we consider two kinds of covariant derivatives $\hat{\nabla}_{\hat{\alpha}}$ and $\tilde{\nabla}_{\hat{\alpha}}$ calculated by means of Christoffel's part of connectivity (6) and the complete anholonomic connectivity (3) correspondingly then matched conditions have to be satisfied. One can prove that the directly under test relation is such a matching condition

$$
\tilde{\nabla}_{\hat{\alpha}} \hat{g}_{\hat{b} \hat{\gamma}}=\hat{\nabla}_{\hat{\alpha}} \hat{g}_{\hat{b} \hat{\gamma}}=0 \text {. }
$$

Let's carry out a further analysis of the relative curvature tensor. Let's consider the expression $0=\hat{\nabla}_{[\hat{\alpha}} \hat{\nabla}_{\hat{\mu}]} \hat{g}_{\hat{\lambda} \hat{v}}=C_{\hat{\mu} \hat{\alpha}}^{\hat{\gamma}} \cdot \frac{\partial \hat{g}_{\hat{\lambda} \hat{v}}}{\hat{\partial} y^{\hat{\gamma}}}-K_{\hat{\alpha} \hat{\mu},(\hat{\lambda} \hat{v})}$.

Using (20) we find

$$
\hat{R}_{\hat{\alpha} \hat{b},(\hat{\gamma} \hat{\mu})}=0 \text {. }
$$

Convoluting (18) on $\hat{\mu}$ and $\hat{\gamma}$ we obtain

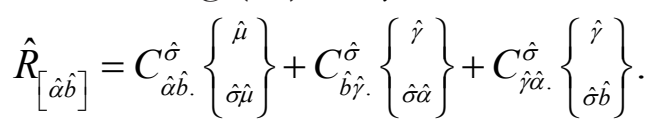

It follows from (24) that the relative Ricci tensor is not symmetrical. Symmetry absence of Ricci tensor is indirectly connected with the expression

$$
\hat{R}_{\hat{\alpha} \hat{b}, \hat{\mu} \hat{\mu}} \neq \hat{R}_{\hat{\gamma} \hat{\mu}, \hat{\alpha} \hat{b}} \text {. }
$$

Let's form a tensor having the same symmetry properties as the usual Riemann-Christoffel tensor. We construct the tensor

$$
\tilde{R}_{\hat{\alpha} \hat{b}, \hat{\mu} \hat{\mu}}=\frac{1}{2}\left(\hat{R}_{\hat{\alpha} \hat{b}, \hat{\gamma} \hat{\mu}}+\hat{R}_{\hat{\gamma} \hat{\mu} \hat{\alpha} \hat{b}}\right),
$$

Using previous formulae one can show that the constructed tensor satisfies the usual Ricci identity

$$
\tilde{R}_{\hat{\alpha} \hat{b}, \hat{\gamma} \hat{\mu}}+\tilde{R}_{\hat{b} \hat{\gamma} \hat{\alpha} \hat{\mu} \hat{\mu}}+\tilde{R}_{\hat{\gamma} \hat{\alpha}, \hat{b} \hat{\mu}}=0,
$$

which results in Ricci tensor symmetry $\tilde{R}_{\hat{\alpha} \hat{b}}$. . We shall point out the equality

$$
\begin{aligned}
& \hat{R}_{\hat{b} \hat{\mu}, \hat{\gamma} \hat{\alpha}}-\hat{R}_{\hat{\gamma} \hat{\alpha}, \hat{\mu} \hat{\mu}}=C_{\hat{\gamma} \hat{b} .}^{\hat{0}} \frac{\hat{\partial} \hat{g}_{\hat{\mu} \hat{\alpha}}}{\hat{\partial} y^{\hat{0}}}+ \\
& +C_{\hat{b} \hat{\alpha} .}^{\hat{0}} \frac{\hat{\partial} \hat{g}_{\hat{\gamma} \hat{\mu}}}{\hat{\partial} y^{\hat{0}}}+C_{\hat{\mu} \hat{\gamma}}^{\hat{0}} \frac{\hat{\partial} \hat{g}_{\hat{b} \hat{\alpha}}}{\hat{\partial} y^{\hat{0}}}+C_{\hat{\alpha} \hat{\mu}}^{\hat{0}} \frac{\hat{\partial} \hat{g}_{\hat{b} \hat{\gamma}}}{\hat{\partial} y^{\hat{0}}} .
\end{aligned}
$$

Calculation of curvature tensor components $\tilde{R}_{\hat{\alpha} \hat{b}, \hat{\mu} \hat{\mu}}$ gives 
$\tilde{R}_{\hat{a} \hat{b}, \hat{q}}=\Omega_{\hat{q} \hat{b}} \Omega_{\hat{a} \hat{c}}-\Omega_{\hat{q} \hat{a}} \Omega_{\hat{b} \hat{c}}-2 \Omega_{\hat{a} \hat{b}} \Omega_{\hat{c} \hat{q}}$,

$\tilde{R}_{\hat{a} \hat{b}, \hat{c} \hat{0}}=2 \hat{\nabla}_{[\hat{a}} \Omega_{\hat{b}] \hat{c}}+2 \Omega_{\hat{a} \hat{b}} F_{\hat{c}}-\frac{1}{2} F_{\hat{b}} \Sigma_{\hat{a} \hat{c}}+\frac{1}{2} F_{\hat{a}} \Sigma_{\hat{c} \hat{b}}$,

$\tilde{R}_{\hat{b} \hat{b}, \hat{c} \hat{c}}=F_{\hat{b}} F_{\hat{c}}-\hat{\nabla}_{(\hat{b}} F_{\hat{c})}-2 \sum_{(\hat{b}}^{\hat{n}} \Omega_{\hat{c}) \hat{n}}+\Omega_{\hat{i} \hat{c}} \Omega_{\hat{b}}^{\hat{n}}$.

Space components of the relative curvature tensor can be represented in the form

$$
\tilde{R}_{\hat{a} \hat{b}, \hat{c} \hat{q}}=\breve{R}_{\hat{a} \hat{b}, \hat{c} \hat{q}}-2 \Sigma_{\hat{q}[\hat{a}} \Sigma_{\hat{b}] \hat{c}} \text {, }
$$

where $\breve{R}_{\hat{a} \hat{b}, \hat{c} \hat{q}}$ is the three-dimensional curvature tensor on the hypersurface orthogonal to world lines of medium particles. This hypersurface is anholonomic when rotations are present. From (29) and (30) we find

$$
\breve{R}_{\hat{a} \hat{b}, \hat{q}}=2 \Sigma_{\hat{q}[\hat{a}} \Sigma_{\hat{b}] \hat{c}}+\Omega_{\hat{q} \hat{b}} \Omega_{\hat{a} \hat{c}}-\Omega_{\hat{q} \hat{a}} \Omega_{\hat{b} \hat{c}}-2 \Omega_{\hat{a} \hat{b}} \Omega_{\hat{c} \hat{q}} .
$$

If there are no rotations the expression (31) passes into one obtained earlier by the author from other considerations [9].

Let's introduce a number of useful identities $\hat{V}_{\hat{\alpha}}=h_{\hat{\alpha}}^{\mu} V_{\mu}=\delta_{\hat{\alpha}}^{\hat{0}}, \tilde{\nabla}_{[\hat{\alpha}} \tilde{\nabla}_{\hat{b}]} \hat{V}_{\hat{\gamma}}=0=$ $=\hat{\nabla}_{[\hat{\alpha}} \tilde{\nabla}_{\hat{b}]} \hat{V}_{\hat{\gamma}}-\mathrm{T}_{[\hat{\alpha} \hat{b}]}^{\hat{\epsilon}]} \tilde{\nabla}_{\hat{\epsilon}} \hat{V}_{\hat{\gamma}}-\mathrm{T}_{[\hat{\alpha}|\hat{\mid}| \hat{\mid} \mid}^{\hat{\epsilon}} \tilde{\nabla}_{\hat{b}]} \hat{V}_{\hat{\epsilon}}$,

$\tilde{\nabla}_{\hat{k}} \hat{V}_{\hat{l}}=\Sigma_{\hat{k} \hat{l}}+\Omega_{\hat{k} \hat{l}}, \tilde{\nabla}_{\hat{0}} \hat{V}_{\hat{l}}=F_{\hat{l}}$, $\tilde{\nabla}_{\hat{k}} \hat{V}_{\hat{0}}=\tilde{\nabla}_{\hat{0}} \hat{V}_{\hat{0}}=0$.

It follows from (32) that

$$
\hat{\nabla}_{[\hat{a}} \Sigma_{\hat{b}]_{\hat{c}}}+\hat{\nabla}_{[\hat{a}} \Omega_{\hat{b}] \hat{c}}=-\Omega_{\hat{a} \hat{b}} F_{\hat{c}} \text {. }
$$

Executing the circular permutation of $\hat{a}, \hat{b}, \hat{c}$ indices in (33) we obtain two additional identities, summing up these ones with (33) we have the identity

$$
\begin{aligned}
& \hat{\nabla}_{\hat{a}} \Omega_{\hat{b} \hat{c}}+\hat{\nabla}_{\hat{b}} \Omega_{\hat{c} \hat{a}}+\hat{\nabla}_{\hat{c}} \Omega_{\hat{a} \hat{b}}+ \\
& +F_{\hat{a}} \Omega_{\hat{b} \hat{c}}+F_{\hat{b}} \Omega_{\hat{c} \hat{a}}+F_{\hat{c}} \Omega_{\hat{a} \hat{b}} \equiv 0 .
\end{aligned}
$$

The similar identity is obtained in [8]. Using expressions (18) and (33) we write (29) in the form

$$
\begin{aligned}
& \tilde{R}_{\hat{a} \hat{b}, \hat{c} \hat{0}}=-\hat{\nabla}_{[\hat{a}} \Sigma_{\hat{b}] \hat{c}}-\frac{1}{2} F_{\hat{b}} \Sigma_{\hat{a} \hat{c}}+\frac{1}{2} F_{\hat{a}} \Sigma_{\hat{c} \hat{b}}, \\
& \tilde{R}_{\hat{0} \hat{b}, \hat{c} \hat{0}}=-\frac{\hat{\partial} \Sigma_{\hat{b} \hat{c}}}{\hat{\partial} y^{\hat{0}}}+\sum_{\hat{c}}^{\hat{n}} \Sigma_{\hat{b} \hat{n}} .
\end{aligned}
$$

It follows from (29) and (35) that for Born rigid irrotational NRF the relative curvature tensor is equal to zero.

For Ricci tensor components we have ${ }^{1}$

$$
\begin{aligned}
& \tilde{R}_{\hat{b} \hat{c}}=-\frac{\hat{\partial} \Sigma_{\hat{b} \hat{c}}}{\hat{\partial} y^{\hat{o}}}+\sum_{\hat{n}}^{\hat{n}} \Sigma_{\hat{b} \hat{c}}+2 \sum_{\hat{c}}^{\hat{n}} \Sigma_{\hat{b} \hat{n}}+\breve{R}_{\hat{b} \hat{c}} \equiv \\
& \equiv F_{\hat{b}} F_{\hat{c}}-\hat{\nabla}_{(\hat{b}} F_{\hat{c})}-2 \sum_{(\hat{b}}^{\hat{n}} \Omega_{\hat{c}) \hat{n}}+\Omega_{\hat{n} \hat{b}} \Omega_{\hat{c}}^{\hat{n}}, \\
& \tilde{R}_{\hat{b} \hat{0}}=-\hat{\nabla}_{\hat{a}} \Omega_{\hat{b}}^{\hat{a}}-2 \Omega_{\hat{a} \hat{b}} F^{\hat{a}}-\frac{1}{2} F_{\hat{a}} \Sigma_{\hat{a} \hat{b}}+\frac{1}{2} F_{\hat{b}} \Sigma_{\hat{c}}^{\hat{c}} \equiv \\
& \equiv \hat{\nabla}_{\hat{a}} \Sigma_{\hat{b}}^{\hat{a}}-\hat{\nabla}_{\hat{b}} \Sigma_{\hat{a}}^{\hat{a}}+\frac{1}{2} F_{\hat{b}} \Sigma_{\hat{a}}^{\hat{a}}-\frac{1}{2} F^{\hat{a}} \Sigma_{\hat{a} \hat{b}},
\end{aligned}
$$

$\tilde{R}_{\hat{0} \hat{0}}=-\frac{\hat{\partial} \Sigma_{\hat{b}}^{\hat{b}}}{\hat{\partial} y^{\hat{0}}}-\Sigma_{\hat{n} \hat{c}} \Sigma^{\hat{\hat{c}} \hat{c}} \equiv$

$\equiv F_{\hat{n}} F^{\hat{n}}-\hat{\nabla}_{\hat{n}} F^{\hat{n}}+\Omega_{\hat{n} \hat{b}} \Omega^{\hat{b} \hat{n}}$.

For the scalar curvature we obtain

$$
\tilde{R}=2 F_{\hat{n}} F^{\hat{n}}-2 \hat{\nabla}_{\hat{n}} F^{\hat{n}}+\Omega_{\hat{n} \hat{b}} \Omega^{\hat{b} \hat{n}} .
$$

We find truncated Bianchi identity from identity (19)

$$
\begin{aligned}
& \hat{\nabla}_{\hat{\alpha}}\left(\tilde{R}^{\hat{\epsilon} \hat{\alpha}}-\frac{1}{2} \hat{g}^{\hat{\epsilon} \hat{\alpha}} \tilde{R}\right)=-\hat{\nabla}_{\hat{\alpha}} \hat{R}^{[\hat{[} \hat{\alpha}]}+ \\
& +2 C_{. \hat{\alpha} \hat{\sigma}}^{\hat{\epsilon}}\left(\hat{R}^{\hat{\alpha} \hat{\alpha}}+\hat{R}^{[\hat{\sigma} \hat{\alpha}]}\right)+C_{\hat{\alpha} \hat{\alpha}, \hat{\sigma}} \hat{R}^{\hat{\epsilon} \hat{\sigma}, \hat{b} \hat{\alpha}} .
\end{aligned}
$$

One can see from expression (38) that the NRF Einstein tensor being in parenthesis in the first member of the equality is essentially differed from the GR Einstein tensor. For that tensor the second member of the equality is identically zero.

${ }^{1}$ We point out that curvature tensors are determined accurate within a mathematical character by different authors. For example, in [10] and [11] curvature tensors coincide and they are differed from the curvature tensor used in this part on the basis of [7] by the sign. The Ricci tensor in [7, 11] and in our case is obtained by means of the convolution on the first and fourth indices and in [10] the convolution is realized on the first and third indices. Therefore Ricci tensors and the scalar curvature in $[7,10]$ and in our case coincide and they are differed from [11] by the sign. 
Let's compare obtained results with A.L. Zel'manov ones [8] introducing for convenience the designations used in [8].

$$
\begin{aligned}
& \hat{g}_{\hat{a} \hat{b}}=-h_{a b}, \quad \sum_{\hat{a} \hat{b}}=-\frac{1}{c} D_{a b}, \quad \Omega_{\hat{c} \hat{\alpha}}=-\hat{A}_{c a}, \\
& \sum_{\hat{a}}^{\hat{a}}=\frac{1}{c} D, \quad \sum_{\hat{c}}^{\hat{n}}=\frac{1}{c} D_{c}^{n}, \quad \Omega_{\hat{c} .}^{\alpha}=\frac{1}{c} A_{c .}^{a}, \\
& F_{\hat{b}}=\frac{1}{c^{2}} F_{b}, \quad F^{\hat{a}}=-\frac{1}{c^{2}} F^{a}, \\
& \frac{\hat{\partial}}{\hat{\partial} y^{\hat{k}}}=\frac{* \partial}{\partial x^{k}}, \quad \frac{\hat{\partial}}{\hat{\partial} y^{\hat{0}}}=\frac{1}{c} \frac{\partial}{\partial t} .
\end{aligned}
$$

Using relations (36) we obtain following identities in Zel'manov designations

$$
\begin{aligned}
& \frac{* \partial D_{i k}}{\partial t}-\left(D_{i j}+A_{i j}\right)\left(D_{k}^{j}+A_{k .}^{j}\right)+D D_{i k}-D_{i j} D_{k}^{j}+ \\
& +3 A_{i j} A_{k .}^{j}+\hat{\nabla}_{(i} F_{k)}-\frac{1}{c^{2}} F_{i} F_{k}+c^{2} \breve{R}_{i k} \equiv 0, \\
& \hat{\nabla}_{j}\left(h^{i j} D-D^{i j}-A^{i j}\right)+\frac{2}{c^{2}} F_{i} A^{i j} \equiv 0, \\
& \frac{* \partial D}{\partial t}+D_{j k} D^{k j}+A_{j k} A^{k j}+\hat{\nabla}_{j} F^{j}-\frac{1}{c^{2}} F_{j} F^{j} \equiv 0 .
\end{aligned}
$$

First members of identities in (39) are Ricci tensor components specifying the first member of Einstein equations in the chronometric invariant theory (CIT). In our case these components are equal to zero. Obtained result is not unexpected. An initial space in which a continuum motion is studied was the flat Minkowski space. The origin of the relative curvature tensor differed from zero is stipulated for the division of zero anholonomic curvature tensor of the flat space-time into two nonzero parts.

If the initial space was Riemannian (as in GR) then the curvature tensor of the initial space prescribed in the anholonomic comoving Lagrangian NRF would add to the first member of equality (8). It had to result in the change of some kinematic identities. In particular in the second member of identity (33) the term will be added

$$
\tilde{\nabla}_{[\hat{a}} \tilde{\nabla}_{\hat{b}]^{\prime}} \hat{V}_{\hat{c}}=-\frac{1}{2} R_{\hat{a} \hat{b}, \hat{c} .}^{\hat{0}} .
$$

Making a convolution on $\hat{a}$ and $\hat{c}$, raising $\hat{b}$ index in the new identity we obtain the expression $R^{\hat{b} \hat{0}}$ of Ricci tensor component in Zel'manov theory.

The purpose of the investigation proposed in this article is the separation of the spacetime curvature contribution stipulated for the noninertiality of the observers moving together with the medium in an arbitrary force field. As the field of 4-velocities $V_{\mu}$ appears as the integration result of the relativistic continuum motion equation in a flat space-time then the expansion

$\nabla_{\mu} V_{v}=\Sigma_{\mu v}+\Omega_{\mu v}+V_{\mu} F_{v}$, saves as mathematical identity.

Although the law of continuum motion in Lagrangian coordinates (1) is holonomic, "space" vectors of affine frames connecting adjacent Lagrangian particles can not appear as a result of differentiation of 4-radius vector $x^{\text {h }}$ on $y^{\hat{k}}$ Lagrangian coordinates, since the hypersurface of simultaneous events is not orthogonal to world lines of medium particles, when a proper time is used as a time parameter. Therefore the nonholonomity object differed from zero arises from the physical demand of an "allocation" of space reference marks on the hypersurface orthogonal to world lines.

Form of the nonholonomity object depends on the selection of a time parameter. For the interval element we find

$d S^{2}=d y^{\hat{0}^{2}}+g_{\mu \theta}^{*} \frac{\partial \Psi^{\mu}}{\partial y^{\hat{n}}} \frac{\partial \Psi^{v}}{\partial y^{\hat{k}}} d y^{\hat{n}} d y^{\hat{k}}$,

$g_{\mu v}^{*}=g_{\mu v}-V_{\mu} V_{v}$.

$g_{\mu v}^{*}$ is the projection operator in Minkowski space projecting tensors on the hypersurface orthogonal to world lines of basis particles

$$
d y^{\hat{0}}=d \xi^{\hat{0}}+V_{\mu} \frac{\partial \Psi^{\mu}}{\partial y^{\hat{n}}} d y^{\hat{n}}=V_{\mu} d x^{\mu} .
$$

One can see from (41) that $d y^{\hat{0}}$ is not a total differential, that is $y^{\hat{0}}$ is the anholonomic coordinate. 
Interval element (40) is equivalent to a fragmentation of four-dimensional interval by two parts in the comoving NRF. One part $d y^{\hat{0}}=V_{\mu} d x^{\mu}$ is the time element of the observer moving together with the medium and other part is the element of three-dimensionalinterval on the hypersurface orthogonal to world lines of medium particles. Similar fragmentation is presented in [8] and [12]. As in the comoving NRF obvious relations are valid

$$
\begin{aligned}
& V^{\hat{k}}=h_{\mu}^{\hat{k}} V^{\mu}=\frac{d y^{\hat{k}}}{d \xi^{\hat{0}}}=0, \\
& V_{\hat{k}}=V_{\mu} \frac{\partial \Psi^{\mu}}{\partial y^{\hat{k}}}=g_{\hat{k} \hat{\alpha}} V^{\tilde{\alpha}}=g_{\hat{k} \hat{0}} V^{\hat{0}}=\frac{g_{\hat{k} \hat{0}}}{\sqrt{g_{\hat{0} \hat{0}}}},
\end{aligned}
$$

then the space interval element in Lagrangian comoving NRF has the form

$$
d l^{2}=\left(\frac{g_{\hat{n} \hat{0}} g_{\hat{k} \hat{0}}}{g_{\hat{0} \hat{0}}}-g_{\hat{n} \hat{k}}\right) d y^{\hat{n}} d y^{\hat{k}} .
$$

The interval element (43) coincides with the well-known relation [10]. We point out that relations (42) and (43) are general and they do not depend on concrete form of Lame parameters (2).

The constructed relative curvature tensor is the tensor relatively anholonomic transformations. It is interesting to construct the relative curvature tensor corresponding to the usual generally covariant RiemannianChristoffel tensor relatively arbitrary holonomic transformations.

In accordance with (6) the nonholonomic connectivity is expanded into the Christoffel's connectivity part and the sum of anholonomity objects. Christoffel's connectivity part is calculated by the metric tensor (9)

$$
\begin{aligned}
& \left\{\begin{array}{c}
\hat{\sigma} \\
\hat{\alpha} \hat{b}
\end{array}\right\}=\frac{1}{2} \hat{g} \hat{\sigma} \hat{\gamma}\left(\hat{\partial}_{\hat{\alpha}} \hat{g}_{\hat{b} \hat{\gamma}}+\hat{\partial}_{\hat{b}} \hat{g}_{\hat{\gamma} \hat{\alpha}}-\hat{\partial}_{\hat{\gamma}} \hat{g}_{\hat{\alpha} \hat{b}}\right), \\
& \hat{\partial}_{\hat{\alpha}} \equiv \frac{\hat{\partial}}{\hat{\partial} y^{\hat{\alpha}}} .
\end{aligned}
$$

Obviously connectivity (44) differs from usual holonomic one, as directional derivatives are present in connectivity (44) instead of partial derivatives. On the basis of the directional derivative determination and using (2) we have

$$
\begin{aligned}
& \hat{\partial}_{\hat{\alpha}}=h_{\hat{\alpha}}^{\mu} \frac{\partial}{\partial x^{\mu}}=\frac{\partial}{\partial y^{\hat{\alpha}}}+L_{\hat{\alpha}} \frac{\partial}{\partial s}, L_{\hat{\alpha}} \equiv \hat{V}_{\hat{\alpha}}-V_{\hat{\alpha}}, \\
& \hat{V}_{\hat{\alpha}}=h_{\hat{\alpha}}^{\mu} V_{\mu}=\delta_{\hat{\alpha}}^{\hat{0}}, V_{\hat{\alpha}}=V_{\mu} \frac{\partial x^{\mu}}{\partial y^{\hat{\alpha}}},
\end{aligned}
$$

where the differentiation on $y^{\hat{0}}$ is equivalent to the differentiation on $\xi^{\hat{0}}$ or on the length $\mathrm{s}$ along basis world lines.

It follows from (45) that $L_{\hat{0}}=0$.

From (6) and (45) we find

$$
\begin{aligned}
& \{\hat{\alpha} \hat{b}, \hat{\gamma}\}=\tilde{\Gamma}_{\hat{\alpha} \hat{b}, \hat{\gamma}}+\tilde{T}_{\hat{\alpha} \hat{b} \hat{\gamma}}, \\
& \tilde{\mathrm{T}}_{\hat{\alpha} \hat{b} \hat{\gamma}}=L_{\hat{b}} \Sigma_{\hat{\gamma} \hat{\alpha}}+L_{\hat{\alpha}} \Sigma_{\hat{\gamma} \hat{b}}-L_{\hat{\gamma}} \Sigma_{\hat{\alpha} \hat{b}}, \\
& \Sigma_{\hat{\gamma} \hat{\alpha}}=\frac{1}{2} \frac{\partial \hat{g}_{\hat{\alpha} \hat{b}}}{\partial s}, \Sigma_{\hat{0} \hat{0}}=\Sigma_{\hat{0} \hat{k}}=0,
\end{aligned}
$$

where $\tilde{\Gamma}_{\hat{\alpha} \hat{b}, \hat{\gamma}}$ is the holonomic Christoffel connectivity calculated in accordance with metric (9). On the basis of the analysis carried out the anholonomic connectivity determined by expansion (6) can be presented in the form $\Gamma_{\hat{\alpha} \hat{b}}^{\hat{\sigma}}=\tilde{\Gamma}_{\hat{\alpha} \hat{b}}^{\hat{\sigma}}+\Pi_{\hat{\alpha} \hat{b}}^{\hat{\sigma}}, \Pi_{\hat{\alpha} \hat{b}}^{\hat{\sigma}}=\mathrm{T}_{\hat{\alpha} \hat{b}}^{\hat{\sigma}}+\tilde{T}_{\hat{\alpha} \hat{b}}^{\hat{\sigma}}$.

Substituting in (7) the anholonomic connectivity $\Gamma_{\hat{\alpha} \hat{b}}^{\hat{\sigma}}$ by the connectivity sum from (47) and taking into account (45) and (6) we obtain the expansion

$$
\begin{aligned}
& R_{\hat{\alpha} \hat{\hat{\gamma}}}^{\ldots}=2 \partial_{[\hat{\alpha}}^{\ldots} \tilde{\Gamma}_{\hat{b}]_{\hat{\gamma}}^{\hat{\mu}}}^{\hat{\mu}}+2 \partial_{[\hat{\alpha}} \Pi_{\hat{b}] \hat{\gamma}}^{\hat{\mu}}+ \\
& +2 L_{[\hat{\alpha}} \frac{\partial \Gamma_{\hat{b}] \hat{\gamma}}^{\hat{\mu}}}{\partial s}+2 \Gamma_{[\hat{\alpha} \hat{\hat{\varepsilon}}|\hat{\hat{b}}| \hat{\hat{b}}] \hat{\gamma}}^{\hat{\hat{\gamma}}}+2 C_{\hat{\alpha} \hat{\beta}}^{\hat{\hat{\beta}}} \Gamma_{\hat{\varepsilon} \hat{\gamma}}^{\hat{\mu}} \equiv 0 .
\end{aligned}
$$

From expansion (48) one can select the term in explicit form

$$
\tilde{K}_{\hat{\alpha} \hat{b} \hat{\gamma}}^{\ldots \hat{\mu}}=2 \partial_{[\hat{\alpha}} \tilde{\Gamma}_{\hat{b}] \hat{y}}^{\hat{\mu}}+2 \tilde{\Gamma}_{[\hat{\alpha} \mid \hat{\varepsilon}}^{\hat{\mu}} \tilde{\Gamma}_{\hat{b}] \hat{\gamma}}^{\hat{\hat{y}}},
$$

which corresponds to usual generally covariant relatively holonomic transformations Riemannian-Christoffel tensor. From (48) and (49) we have

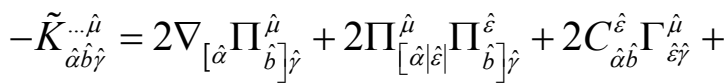

$$
\begin{aligned}
& +2 L_{[\hat{\alpha}} \frac{\partial \Gamma_{\hat{b}] \hat{\gamma}}^{\hat{\mu}}}{\partial s} \text {. }
\end{aligned}
$$


It is necessary to make following remarks concerning formula (50):

1. Covariant derivative in the second member of (50) is calculated in an ordinary way (as for a tensor) in accordance with the holonomic Christoffel connectivity from $\Pi_{\hat{\alpha} \hat{b}}^{\hat{\sigma}}$ object. This object is not a tensor relatively holonomic transformations.

2. All values including into the second member of equality (identity) (50) also are not tensors relatively holonomic transformations. However their combination is a generally covariant tensor.

Thus, in the Lagrangian comoving reference frame one can introduce three connectivities in a general coordination: absolute anholonomic connectivity $\Gamma_{\hat{\varepsilon} \hat{\gamma}}^{\hat{\mu}}$, calculated in accordance with (3), relative anholonomic connectivity $\left\{\begin{array}{c}\hat{\sigma} \\ \hat{\alpha} \hat{b}\end{array}\right\}$, specified by means of expansion (6) and rêative holonomic connectivity $\tilde{\Gamma}_{\hat{\alpha} \hat{b}, \hat{\gamma}}$, obtained from expansion (46).

It is clear that it is possible only in the case if covariant derivatives from the metric tensor (9) for each connectivity are equal to zero. Let us prove that it is right. Using formulae (3), (9) and substituting them into the expression

$$
\tilde{\nabla}_{\hat{\mu}} \hat{g}_{\hat{\alpha} \hat{b}}=\hat{\partial}_{\hat{\mu}} \hat{g}_{\hat{\alpha} \hat{b}}-\Gamma_{\hat{\mu} \hat{\alpha}}^{\hat{v}} \hat{g}_{\hat{v} \hat{b}}-\Gamma_{\hat{\mu} \hat{b}}^{\hat{v}} \hat{g}_{\hat{\alpha} \hat{v}},
$$

we ascertain that $\tilde{\nabla}_{\hat{\mu}} \hat{g}_{\hat{\alpha} \hat{b}} \equiv 0$.

Using expansion (6) by means of (51) we find

$$
\hat{\nabla}_{\hat{\mu}} \hat{g}_{\hat{\alpha} \hat{b}}=\tilde{\nabla}_{\hat{\mu}} \hat{g}_{\hat{\alpha} \hat{b}}+\mathrm{T}_{\hat{\mu} \hat{\alpha}}^{\hat{v}} \hat{g}_{\hat{v} \hat{b}}+\mathrm{T}_{\hat{\mu} \hat{b}}^{\hat{v}} \hat{g}_{\hat{\alpha} \hat{v}} \text {. }
$$

As in the last formula the sum of two connectivity affine deformation tensors ${ }^{2}$ forms zero, then $\hat{\nabla}_{\hat{\mu}} \hat{g}_{\hat{\alpha} \hat{b}}=\tilde{\nabla}_{\hat{\mu}} \hat{g}_{\hat{\alpha} \hat{b}}=0$.

Finally from the equality

${ }^{2}$ We point out that unlike $\Pi_{\hat{b} \hat{\gamma}}^{\hat{\mu}}$ objects $\mathrm{T}_{\hat{b} \hat{\gamma}}^{\hat{\mu}}$ are the tensors relatively anholonomic transformations. To prove that it is enough to calculate covariant derivatives of an arbitrary vector on connectivities

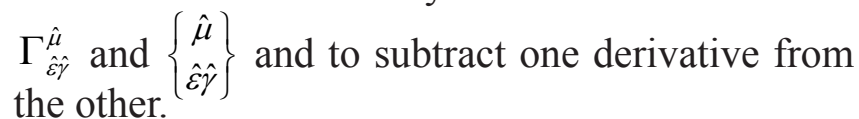

$$
\begin{aligned}
& \nabla_{\hat{\mu}} \hat{g}_{\hat{\alpha} \hat{b}}=\hat{\nabla}_{\hat{\mu}} \hat{g}_{\hat{\alpha} \hat{b}}+\mathrm{T}_{\hat{\mu} \hat{\alpha}, \hat{b}}^{*}+\mathrm{T}_{\hat{\mu} \hat{b}, \hat{\alpha}}^{*}-2 L_{\hat{\mu}} \Sigma_{\hat{\alpha} \hat{b}}, \\
& \mathrm{~T}_{\hat{\mu} \hat{\alpha}, \hat{b}}^{*}=L_{\hat{\mu}} \Sigma_{\hat{\alpha} \hat{b}}+L_{\hat{\alpha}} \Sigma_{\hat{\mu} \hat{b}}-L_{\hat{b}} \Sigma_{\hat{\mu} \hat{\alpha}}
\end{aligned}
$$

it follows that

$$
\mathrm{T}_{\hat{\mu} \hat{\alpha}, \hat{b}}^{*}+\mathrm{T}_{\hat{\mu} \hat{b}, \hat{\alpha}}^{*}-2 L_{\hat{\mu}} \Sigma_{\hat{\alpha} \hat{b}}=0 .
$$

Therefore we have

$$
\tilde{\nabla}_{\hat{\mu}} \hat{g}_{\hat{\alpha} \hat{b}}=\hat{\nabla}_{\hat{\mu}} \hat{g}_{\hat{\alpha} \hat{b}}=\nabla_{\hat{\mu}} \hat{g}_{\hat{\alpha} \hat{b}}=0 \text {. }
$$

The mathematical apparatus developed in this paragraph describing properties of Lagrangian co-moving reference frames with prescribed law of motion (1) proposed that the proper time appeared in (1) as a time parameter. However often in describing the transition from IRF to NRF other time parameter is used, for example, the IRF time.

Therefore it is interesting to develop such an apparatus which is suitable for an arbitrary time parameter. We shall consider in (1) that $\xi^{\hat{0}}$ is the arbitrary time parameter. For 4-velocity $V^{\mu}$ in Lagrangian variables the relation is valid

$V^{\mu}=\Theta \frac{\partial \Psi^{\mu}}{\partial \xi^{\hat{0}}}$

where $\Theta$ multiplier is determined from the normalizing condition of 4-velocity per unit.

$$
\Theta^{2}=\frac{1}{g_{\mu \nu} \frac{\partial \Psi^{\mu}}{\partial \xi^{\hat{0}}} \frac{\partial \Psi^{v}}{\partial \xi^{\hat{0}}} .}
$$

Corresponding Lamé coefficients have the form

$$
\begin{aligned}
& h_{\hat{k}}^{\mu}=\left(\delta_{\varepsilon}^{\mu}-V^{\mu} V_{\varepsilon}\right) \frac{\partial \Psi^{\varepsilon}}{\partial y^{\hat{k}}}, h_{\hat{0}}^{\mu}=\frac{\partial \Psi^{\mu}}{\partial \xi^{\hat{0}}}=\frac{V^{\mu}}{\Theta}, \\
& h_{\mu}^{\hat{k}}=\frac{\partial y^{\hat{k}}}{\partial x^{\mu}} \rightarrow h_{\mu}^{\hat{0}}=\Theta V_{\mu} .
\end{aligned}
$$

One can represent connectivity coefficients $\Gamma_{\hat{\alpha} \hat{b}}^{\hat{\sigma}}$ in Minkowski space for anholonomic coordinates in the form (3) and expansion (6). We form the nonholonomity object $C_{\hat{\alpha} \hat{b}}^{\hat{\sigma}}$ (5) from Lamé coefficients. Concrete form of the nonholonomity object depends on selected 
Lamé coefficients which are determined depending on the time parameter along world lines of basis particles. For case (58) we find

$$
C_{\hat{k} \hat{l}}^{\hat{0}}=\Theta \Omega_{\hat{k} \hat{l}}, 2 C_{\hat{0} \hat{k}}^{\hat{0}}=F_{\hat{k}}-\frac{\hat{\partial} \ln \Theta}{\hat{\partial} y^{\hat{k}}}, C_{\hat{\alpha} \hat{b}}^{\hat{k}}=0,
$$

where

$$
\Omega_{\hat{k} \hat{l}}=\Omega_{\mu \nu} h_{\hat{k}}^{\mu} h_{\hat{l}}^{v}, F_{\hat{k}}=F_{\mu} h_{\hat{k}}^{\mu} .
$$

Metric coefficients for Lamé parameters (58) have the form

$$
\hat{g}_{\hat{\alpha} \hat{b}}=g_{\mu v} h_{\hat{\alpha}}^{\mu} h_{\hat{b}}^{v}, \hat{g}_{\hat{0} \hat{0}}=\frac{1}{\Theta^{2}}, \hat{g}_{\hat{0} \hat{k}}=0,
$$

where $g_{\mu \nu}$ is the metric tensor in Eulerian coordinates of Minkowski space. Christoffel symbols are calculated in the usual way with substitution of partial derivatives by directional derivatives, and $\hat{\nabla}_{\hat{\alpha}}$ operator is calculated by means of Christoffel connectivity. Commutation relations for directional derivatives are determined by general formulae (10)

$$
\begin{aligned}
& \frac{\hat{\partial}^{2}}{\hat{\partial} y^{\hat{k}} \hat{\partial} y^{\hat{l}}}-\frac{\hat{\partial}^{2}}{\hat{\partial} y^{\hat{\imath}} \hat{\partial}^{\hat{k}}}=2 \Theta \Omega_{\hat{l} \hat{k}} \frac{\hat{\partial}}{\partial \hat{\partial}^{\hat{0}}}=2 \Omega_{\hat{l}} \frac{\partial}{\partial s}, \\
& \frac{\hat{\partial}^{2}}{\hat{\partial} y^{\hat{k}} \hat{\partial} y^{\hat{0}}}-\frac{\hat{\partial}^{2}}{\hat{\partial} y^{\hat{0}} \hat{\partial} y^{\hat{k}}}=\left(F_{\hat{k}}-\frac{\hat{\partial} \ln \Theta}{\hat{\partial} y^{\hat{k}}}\right) \frac{\hat{\partial}}{\hat{\partial} y^{\hat{0}}}= \\
& =\frac{1}{\Theta}\left(F_{\hat{k}}-\frac{\partial \ln \Theta}{\hat{\partial} y^{\hat{k}}}\right) \frac{\partial}{\partial s} .
\end{aligned}
$$

For tensor components of the connectivity affine deformation we find

$$
\begin{aligned}
& \mathrm{T}_{\hat{0} \hat{k}, \hat{0}}=-\left(F_{\hat{k}}-\frac{\hat{\partial} \ln \Theta}{\hat{\partial} y^{\hat{k}}}\right) \frac{1}{\Theta^{2}}, \\
& \mathrm{~T}_{\hat{0} \hat{0}, \hat{k}}=\left(F_{\hat{k}}-\frac{\hat{\partial} \ln \Theta}{\hat{\partial} y^{\hat{k}}}\right) \frac{1}{\Theta^{2}}, \\
& \mathrm{~T}_{\hat{m} \hat{l}, \hat{k}}=\mathrm{T}_{\hat{k} \hat{0}, \hat{0}}=0, \mathrm{~T}_{\hat{0} \hat{l}, \hat{k}}=\mathrm{T}_{\hat{\imath} \hat{0}, \hat{k}}=\frac{1}{\Theta} \Omega_{\hat{\imath} \hat{k}}, \\
& \mathrm{~T}_{\hat{k} \hat{l}, \hat{0}}=-\frac{1}{\Theta} \Omega_{\hat{k} \hat{l}} .
\end{aligned}
$$

Construction of the relative anholonomic and holonomic curvature tensor is executed in accordance with the same rules as earlier. Directional derivatives are connected with partial derivatives by the formula

$$
\begin{aligned}
& \hat{\partial}_{\hat{\alpha}}=h_{\hat{\alpha}}^{\mu} \frac{\partial}{\partial x^{\mu}}=\frac{\partial}{\partial y^{\hat{\alpha}}}+L_{\hat{\alpha}} \frac{\partial}{\partial \xi^{\hat{0}}}, \\
& L_{\hat{\alpha}} \equiv\left(\delta_{\hat{\alpha}}^{\hat{0}}-\Theta V_{\hat{\alpha}}\right), V_{\hat{\alpha}}=V_{\mu} \frac{\partial x_{\mu}}{\partial y^{\hat{\alpha}}} .
\end{aligned}
$$

In accordance with (6) the anholonomic connectivity is expanded into the Christoffel connectivity part and the sum of nonholonomity objects. Christoffel connectivity part is calculated by the metric tensor (9) in accordance with formula (44). Obviously, that connectivity (44) differs from usual holonomic connectivity. In connectivity (44) directional derivatives are used instead of partial derivatives. On the basis of the determination of the directional derivative using (2) and (64) we find

$$
\begin{aligned}
& \{\hat{\alpha} \hat{b}, \hat{\gamma}\}=\tilde{\Gamma}_{\hat{\alpha} \hat{b}, \hat{\gamma}}+\tilde{T}_{\hat{\alpha} \hat{b} \hat{\gamma}}, \\
& \tilde{\mathrm{T}}_{\hat{\alpha} \hat{b} \hat{\gamma}}=\frac{1}{\Theta}\left[L_{\hat{b}} \Sigma_{\hat{\gamma} \hat{\alpha}}+L_{\hat{\alpha}} \Sigma_{\hat{\gamma} \hat{b}}-L_{\hat{\gamma}} \Sigma_{\hat{\alpha} \hat{b}}\right], \\
& \Sigma_{\hat{\gamma} \hat{\alpha}}=\frac{1}{2} \Theta \frac{\partial \hat{g}_{\hat{\alpha} \hat{b}}}{\partial \xi^{\hat{0}}}, \Sigma_{\hat{0} \hat{0}}=\Sigma_{\hat{0} \hat{k}}=0,
\end{aligned}
$$

where $\tilde{\Gamma}_{\hat{\alpha} \hat{b}, \hat{\gamma}}$ is the holonomic Christoffel connectivity calculated in accordance with metric (61) for which we have

$$
\begin{aligned}
& \tilde{\Gamma}_{\hat{0}, \hat{l} \hat{l}}=-\frac{1}{2} \frac{\partial \hat{g}_{\hat{k} \hat{l}}}{\partial \xi^{\hat{0}}}, \tilde{\Gamma}_{\hat{0}, \hat{0} \hat{l}}=\frac{1}{2} \frac{\partial \hat{g}_{\hat{0} \hat{0}}}{\partial y^{\hat{l}}}, \tilde{\Gamma}_{\hat{0}, \hat{0} \hat{0}}=\frac{1}{2} \frac{\partial \hat{g}_{\hat{0} \hat{0}}}{\partial y^{\hat{0}}}, \\
& \tilde{\Gamma}_{\hat{n}, \hat{0} \hat{l}}=\frac{1}{2} \frac{\partial \hat{g}_{\hat{n} \hat{l}}}{\partial y^{\hat{0}}}, \tilde{\Gamma}_{\hat{n}, \hat{0} \hat{0}}=-\frac{1}{2} \frac{\partial \hat{g}_{\hat{0} \hat{0}}}{\partial y^{\hat{n}}}, \tilde{\Gamma}_{\hat{k} \hat{\imath}}^{\hat{n}}=\tilde{\Lambda}_{\hat{k} \hat{l}}^{\hat{n}},
\end{aligned}
$$

where $\tilde{\Lambda}_{\hat{k} \hat{\imath}}^{\hat{n}}$ are the three-dimensional Christoffel symbols formed from three-dimensional tensor $\hat{\gamma}_{\hat{k} \hat{l}}=-\hat{g}_{\hat{k} \hat{l}}$.

The relative holonomic curvature tensor can be calculated in accordance with (49) or (50). The calculation in accordance with (49) results in relations 


$$
\begin{aligned}
& -\tilde{K}_{\hat{0} \hat{k}, \hat{l} \hat{m}}=\sqrt{\hat{g}_{\hat{0} \hat{0}}}\left(\nabla_{\hat{m}} \Sigma_{\hat{k} \hat{l}}-\nabla_{\hat{l}} \Sigma_{\hat{k} \hat{m}}\right) \text {, } \\
& \tilde{K}_{\hat{i} \hat{k}, \hat{m} \hat{m}}=P_{\hat{i} \hat{k}, \hat{m} \hat{m}}-\left(\sum_{\hat{k} \hat{l}} \sum_{\hat{i} \hat{m}}-\sum_{\hat{k} \hat{m}} \sum_{\hat{i} \hat{l}}\right) \text {, } \\
& -\tilde{K}_{\hat{0} \hat{k}, \hat{0} \hat{m}}=-\hat{g}_{\hat{0} \hat{0}}\left(\frac{\partial \Sigma_{\hat{k} \hat{m}}}{\partial s}-\hat{g}^{\hat{q} \hat{r}} \Sigma_{\hat{k} \hat{q}} \Sigma_{\hat{m} \hat{r}}\right)- \\
& -\frac{1}{2}\left[\frac{\partial^{2} \hat{g}_{\hat{0} \hat{0}}}{\partial y^{\hat{k}} \partial y^{\hat{m}}}-\frac{1}{2} \hat{g}^{\hat{0} \hat{0}} \frac{\partial \hat{g}_{\hat{0} \hat{0}}}{\partial y^{\hat{l}}} \frac{\partial \hat{g}_{\hat{0} \hat{0}}}{\partial y^{\hat{m}}}-\Lambda_{\hat{k} \hat{m}}^{\hat{n}} \frac{\partial \hat{g}_{\hat{0} \hat{0}}}{\partial y^{\hat{n}}}\right] \text {. }
\end{aligned}
$$

It follows from (65) for Born rigid motions (that is at $\Sigma_{\hat{\alpha} \hat{b}}=0$ ) holonomic and anholonomic Christoffel symbols coincide, however relative anholonomic and holonomic curvature tensors are differed from each other due to the anholonomic addition (see (20)).

\section{ELEMENT SQUARE OF RELATIVE CONTINUUM INTERVAL IN LAGRANGIAN COORDINATES}

For example, we shall consider the motion of classic rigid body with the law of motion

$$
x^{a}=\int_{0}^{t} v(\tau) d \tau+y^{a}, x^{0}=c t=\xi^{\hat{0}} .
$$

Here the IRF time is selected as a time parameter $\xi^{\hat{0}}$. Element of the relative interval can be constructed from metric coefficients (61)

$$
\begin{aligned}
& d \tilde{S}^{2}=\frac{1}{\Theta^{2}} d \xi^{\hat{0}^{2}}+g_{\mu \nu}^{*} \frac{\partial \Psi^{\mu}}{\partial y^{\hat{n}}} \frac{\partial \Psi^{v}}{\partial y^{\hat{k}}} d y^{\hat{n}} d y^{\hat{k}}, \\
& g_{\mu \nu}^{*}=g_{\mu \nu}-V_{\mu} V_{v} .
\end{aligned}
$$

$g_{\mu \nu}^{*}$ is the projection operator in Minkowski space projecting the tensors on the hypersurface orthogonal to world lines of basis particles.

The relative interval element $d \tilde{S}^{2}$ differs from the absolute interval element (40) by the normalizing factor before the time coefficient. And, that is more important, that the holonomic element $d \xi^{\hat{0}}=c d t$ is included instead of the anholonomic element $d y^{\hat{0}}$ determined from (41). It is clear that the magnitudes of absolute and relative intervals are not equal. Use of the metric for absolute interval obtained by means of nonholonomic transformations results in zero nonholonomic curvature tensor. Nonholonomic and holonomic curvature tensors differed from zero are obtained from zero nonholonomic curvature tensor by means of the procedure developed above.

Normalizing factor $1 / \Theta^{2}$ can be calculated in accordance with (57). Use of the law of motion (66) results in the form of the interval element square (66)

$$
d \tilde{S}^{2}=\frac{1}{V_{0}^{2}} d x^{0^{2}}-\left(\delta_{n k}+V_{n} V_{k}\right) d y^{\hat{n}} d y^{\hat{k}} .
$$

Relation (68) was obtained earlier by V.I. Rodichev [13], in this relation Eulerian coordinates $X^{\mathrm{k}}$ were included instead of Lagrangian coordinates $y^{k}$ and the motion was not considered as certainly classic rigid.

For particular case when rigid box having at infinity zero velocity falls from infinity at the centrosymmetrical Sun field, the similar formula for the interval (68) is presented in known A. Sommerfeld book [14] with the reference to the unpublished Lents work. Based on this formula Sommerfeld got the interval in the Schwarzchild form using the Newton's law as a first approximation.

Interval (68) in the Rodichev form can be obtained from the interval (67) and for the arbitrary law of continuum motion

$$
x^{a}=\Psi^{a}\left(y^{\hat{k}}, x^{0}\right), x^{0}=c t=\xi^{\hat{0}},
$$

if one introduces the following designation [15]

$$
\frac{\partial \Psi^{k}}{\partial y^{\hat{n}}} d y^{\hat{n}}=d X^{k},
$$

which means that the element connecting two close Lagrangian particles is considered in Euler coordinates at the fixed instant of time $t$. Such method is used in the classical mechanics of continua when deriving the deformation tensor in Lagrangian co-moving reference frame $(\mathrm{RF})$.

For arbitrary continuum motion (69) the relative interval element in Lagrangian coordinates has the form

$$
d \tilde{S}^{2}=\frac{1}{V_{0}^{2}} d \xi^{\hat{0}^{2}}-\left(\delta_{m n}+V_{m} V_{n}\right) \frac{\partial \Psi^{m}}{\partial y^{\hat{l}}} \frac{\partial \Psi^{n}}{\partial y^{\hat{k}}} d y^{\hat{l}} d y^{\hat{k}} .
$$


Let us consider some particular RF cases realized by means of the law of motion (69) and metric (71).

\subsection{UNIFORMLY ROTATING RF}

Unlike the relativistic rigid NRF considered in previous part and realized in the Riemannian space-time we shall follow to the standard transition method [10].

We select the rest-frame in which we introduce cylindrical coordinates $r_{0}, \varphi_{0}, z_{0}, t_{0}$ and move to the rotating $\mathrm{RF} r, \varphi, z, t$ in accordance with formulae:

$$
r_{0}=r, \varphi_{0}=\varphi+\Omega t, z_{0}=z, t_{0}=t \text {, }
$$

where the rotational speed $\Omega$ relatively $z$ axis is considered as constant.

Passing on from Galilean coordinates (71) to cylindrical coordinates we obtain the expression for the relative interval in rotating NRF. The interval element has the form

$$
d \tilde{S}^{2}=\left(1-\frac{\Omega^{2} r^{2}}{c^{2}}\right) c^{2} d t^{2}-d r^{2}-\frac{r^{2} d \phi^{2}}{1-\frac{\Omega^{2} r^{2}}{c^{2}}}-d z^{2} .
$$

To compare we present the range of interval in the standard consideration

$$
\begin{aligned}
& d S^{2}=\left(1-\frac{\Omega^{2} r^{2}}{c^{2}}\right) c^{2} d t^{2}-2 \Omega r^{2} d \phi d t- \\
& -d z^{2}-r^{2} d \phi^{2}-d r^{2} .
\end{aligned}
$$

Both formulas are correct if $r \Omega / c<1$ and satisfy to the stiffness criterion both classic and Born relativistic one. However there is the significant difference between the metrics: metric (72) is realized in the Riemannian space-time and metrics (73) is realized in the flat Minkowski space. At $t=$ const metric (72) corresponds to the element of the "physical" space interval in the rotating reference frame in accordance with (43). Unlike (73) in (72) $g_{0 \mathrm{k}}$ components of metric tensor are absent, this means the possibility of watches synchronization along any closed circuit [10].

The connection between the true time $\tau$ and the time of Minkowski space $t$ is identical for both metrics $d \tau^{2}=\left(1-\frac{\Omega^{2} r^{2}}{c^{2}}\right) d t^{2}$.

Unlike the relativistic rigid NRF both metrics are correct only for finite distances from the rotation axis.

Metrics (72) permits a simple geometrical interpretation.

The relative interval element as well as in IRF in Cartesian coordinates is determined in accordance with Pythagoras theorem for the pseudo-Riemannian space-time: the squared element of the "physical" length is subtracted from the squared proper time (multiplied by the squared light velocity).

\subsection{Relativistic (NONRIgid) UNIfORMLy ACCELERATED NRF}

In Minkowski space there is no any law of motion which results in simultaneous fulfillment of two conditions: relativistic rigidity and uniformly acceleration [1-5]. Therefore we consider as the uniformly accelerated RF the motion of the charged dust in the constant electric field resulting in Logunov metric $d S^{2}=\left(d x^{0}\right)^{2}-\left(d x^{1}\right)^{2}-\left(d x^{2}\right)^{2}-\left(d x^{3}\right)^{2}$, where $x_{0}=c t, x^{1}, x^{2}, x^{3}$ are the Cartesian coordinates, and the law of continuum motion for the Logunov metric has the form

$$
\begin{aligned}
& x^{1}\left(y^{1}, t\right)=y^{1}+\left(c^{2} / a_{0}\right)\left[\sqrt{1+a_{0}^{2} t^{2} / c^{2}}-1\right], \\
& x^{2}=y^{2}, x^{3}=y^{3}, x^{0}=y^{0}
\end{aligned}
$$

or

$$
x^{1}\left(y^{1}, \tau\right)=y^{1}+c^{2} / a_{0}\left[\cosh \left(a_{0} \tau / c\right)-1\right],
$$$$
x^{2}=y^{2}, x^{3}=y^{3}, t=(c / a) \sinh \left(a_{0} \tau / c\right),
$$

where the IRF time is used as a time parameter, and $\tau$ is the proper time

$$
\begin{aligned}
& d S^{2}=\frac{c^{2} d t^{2}}{1+a_{0}^{2} t^{2} / c^{2}}-2 \frac{a_{0} t d t d y^{1}}{\left(1+a_{0}^{2} t^{2} / c^{2}\right)^{1 / 2}}- \\
& -\left(d y^{1}\right)^{2}-\left(d y^{2}\right)^{2}-\left(d y^{3}\right)^{2} \\
& d S^{2}=c^{2}(d \tau)^{2}-2 \sinh \left(a_{0} \tau / c\right) c d \tau d y^{1}- \\
& -\left(d y^{1}\right)^{2}-\left(d y^{2}\right)^{2}-\left(d y^{3}\right)^{2}
\end{aligned}
$$


Substitution of the law of motion into (71) $\left(y^{\hat{k}} \rightarrow y^{k}\right)$ results in the interval element in the form

$$
\begin{aligned}
& d \tilde{S}^{2}=\frac{c^{2} d t^{2}}{1+a_{0}^{2} t^{2} / c^{2}}-\left(1+a_{0}^{2} t^{2} / c^{2}\right)\left(d y^{1}\right)^{2}+ \\
& +\left(d y^{2}\right)^{2}+\left(d y^{3}\right)^{2} .
\end{aligned}
$$

Substitution of the law of motion into (71) (with the substitution $\Theta=1, t \rightarrow \tau$ ) results in the squared interval in the form

$$
\begin{aligned}
& d \tilde{S}^{2}=c^{2} d \tau^{2}-\cosh ^{2}\left(\frac{a_{0} \tau}{c_{2}}\right)\left(d y^{1}\right)^{2}+ \\
& +\left(d y^{2}\right)^{2}+\left(d y^{3}\right)^{2} .
\end{aligned}
$$

Formula (75) was obtained by V.I. Rodichev from other considerations [13] but in Euler coordinates (not Lagrange coordinates as in our case). It is clear that (76) can be obtained from (75) by means of the simple time transformation which is determined by the equality $t=\left(c / a_{0}\right) \sinh \left(a_{0} \tau / c\right)$.

The analysis of formulae (75) and (76) and the comparison with the similar Logunov's relations shows that the elements of the relative intervals (as in the rotary motion case) are calculated in accordance with the Pythagoras theorem for the pseudo-Riemann space, when the squared element of the "physical" length is subtracted from the squared proper time (multiplied by the squared light velocity).

The calculation of the relative curvature tensor for metric (76) results in one component differed from zero

$$
\hat{R}_{\hat{0} \hat{1}, \hat{1}}^{\hat{0}}=-\frac{a_{0}^{2}}{c^{4}} \cosh \left(\frac{a_{0}^{2} \xi^{\hat{0}}}{c^{2}}\right) \text {. }
$$

For the scalar curvature we obtain

$$
\hat{R}=-2 \frac{a_{0}^{2}}{c^{4}} \text {. }
$$

In accordance with the standard viewpoint [10] the synchronization of the watches being at the same physical conditions that is at the same distance from the rotation axis is absent.
In our consideration for therelative interval such "paradox" does not arise.

In considering the rotary motion the watches being at the similar distance from the rotation axis have to show the same proper time. A mathematical transition to the relative interval can be realized by the elementary way: using $d y^{\hat{0}}$ in metric (40) at the fixed value of the Lagrangian particle coordinate $y^{\hat{k}}$ in (41). That results in the interval

$$
d \tilde{S}^{2}=d \xi^{\hat{0}^{2}}+g_{\mu \nu}^{*} \frac{\partial \Psi^{\mu}}{\partial y^{\hat{n}}} \frac{\partial \Psi^{v}}{\partial y^{\hat{k}}} d y^{\hat{n}} d y^{\hat{k}}, g_{\mu \nu}^{*}=g_{\mu \nu}-V_{\mu} V_{\nu},
$$

the length of which in the general case is not equal to the interval length (40).

Thus, from our viewpoint the NRF observer interval (that is the relative interval (78)) is differed from the IRF observer interval using NRF coordinates (40). It follows from the developed mathematical apparatus permitting to select the holonomic curvature tensor from zero anholonomic one, that usual RiemannianChristoffel tensor calculated from the relative interval metric in the general case is differed from zero.

\section{ACCELERATION ADDITION LAW, RELATIVE NRF CURVATURE TENSOR IN MINKOWSKI SPACE}

One can ascertain the physical meaning of the relative curvature tensor on the basis of the analysis of a particle motion in an arbitrary NRF force field.

Let the continuum moves in Minkowski space in some force field. The field of 4-velocity of the medium in Euler variables is $V^{\mu}$. At the same space in the other force field the particle moves with 4-velocity $U^{\mu}$ not coinciding with $V^{\mu}$.

The transition to NRF is realized by means of Lame parameters (2) using the equation of motion

$h_{\alpha}^{\hat{\mu}} \frac{d U^{\alpha}}{d \tilde{S}}=\frac{1}{m_{0} c} h_{\alpha}^{\hat{\mu}} f^{\alpha}$. 
In formula (79) $m_{0}$ is the rest mass of the particle, $f^{\alpha}$ is the 4-force. From (2)-(6) using the equalities

$$
\hat{\nabla}_{\hat{\mu}} h_{\hat{\alpha}}^{v}=h_{\hat{\gamma}}^{v} \mathrm{~T}_{\hat{\mu} \hat{\alpha} .}^{\hat{\gamma}}, \tilde{\nabla}_{\hat{\mu}} h_{\hat{\alpha}}^{v}=0 \text {, }
$$

after simple transformations we obtain

$$
\frac{d \hat{U}^{\hat{\mu}}}{d \tilde{S}}+\left\{\begin{array}{c}
\hat{\mu} \\
\hat{\alpha} \hat{b}
\end{array}\right\} \hat{U}^{\hat{\alpha}} \hat{U}^{\hat{b}}=\frac{1}{m_{0} c} f^{\hat{\mu}}-\mathrm{T}_{\hat{\alpha} \hat{b} .}^{\hat{\mu}} \hat{U}^{\hat{\alpha}} \hat{U}^{\hat{b}} .
$$

If the particle under consideration belongs to one of the NRF basis particles then $U^{\mu}=V^{\mu}$ and it follows from (14) that the second member of equation (81) vanishes. In other words for comoving NRF observers the relative first curvature vectors of basis particle world lines are equal to zero, and the relative space-time curvature is differed from zero. We rewrite equations (81) into components using obvious relation

$$
d \tilde{S}^{2}=d y^{\hat{0}^{2}}-d l^{2}=\left(1-\frac{u^{2}}{c^{2}}\right) d y^{\hat{0}^{2}},
$$

where $u$ is the value of the relative particle velocity. Using expressions (14) and designations (38a) we obtain the motion equations relatively $\mathrm{NRF}$ in the form convenient for the comparison with the Zel'manov work [8]

$$
\begin{aligned}
& \frac{d E}{d \tau}+m D_{\hat{i} \hat{k}} u^{\hat{i}} u^{\hat{k}}-m F_{\hat{i}} u^{\hat{i}}=c^{2} V_{\mu} f^{\mu} \sqrt{1-\frac{u^{2}}{c^{2}}}, \\
& \frac{d p^{\hat{k}}}{d \tau}+\lambda_{\hat{n} \hat{l}}^{\hat{k}} p^{\hat{n}} u^{\hat{l}}+2 m\left(D_{\hat{i}}^{\hat{k}}+A_{\hat{i}}^{\hat{k}}\right) u^{\hat{i}}-m F^{\hat{k}}= \\
& =c f^{\hat{k}} \sqrt{1-\frac{u^{2}}{c^{2}}} .
\end{aligned}
$$

The following designations are introduced in formulae (83) and (84):

$$
E=\frac{m_{0} c^{2}}{\sqrt{1-\frac{u^{2}}{c^{2}}}}, p^{\hat{i}}=\frac{m_{0} u^{\hat{i}}}{\sqrt{1-\frac{u^{2}}{c^{2}}}}, m=\frac{E}{c^{2}}, d \tau=\frac{d y^{\hat{0}}}{c},
$$

where $E$ is the relative (chronometrically invariant (c.i.) [8]) particle energy, $m$ is the relative (c.i.) mass, and $p^{\hat{i}}$ is the relative (c.i.) pulse. The left-hand members of equalities (83) and (84) are identical to the left-hand members of equalities in world motion equations [8]. One can easy prove the equality of the righthand members. Really for the holonomic frames obtained from (1) in the comoving $\mathrm{NRF}$ the equality is valid

$$
\begin{aligned}
& V_{\mu} f^{\mu}=\hat{f}^{\hat{0}}=V_{\hat{0}} f^{\hat{0}}+V_{\hat{k}} f^{\tilde{k}}= \\
& =\sqrt{g_{\hat{0} \hat{0}}} f^{\hat{0}}+\frac{g_{\hat{0} \hat{k}}}{\sqrt{g_{\hat{0} \hat{0}}}} f^{\hat{k}}=g_{\hat{0} \hat{\alpha}} f^{\hat{\alpha}}=\frac{f_{\hat{0}}}{\sqrt{g_{\hat{0} \hat{0}}}},
\end{aligned}
$$

use of this equality proves the coincidence of the right-hand members of equations (83) and work [8]. The proof for (84) is similar. Thus, in spite of the difference of our transition method to NRF and the T.C.I. method, the motion equations relatively NRF coincide.

One can write equation (82) after the convolution with $h_{\hat{\mu}}^{\alpha}$ in Minkowski space in the form covariant relatively arbitrary holonomic transformations of Eulerian coordinates. Using (14) after simple transformations we obtain

$h_{\hat{\mu}}^{\alpha} \frac{\hat{D} \hat{U}^{\hat{\mu}}}{d \tilde{S}} \equiv K^{\alpha}=\frac{f^{\alpha}}{m_{0} c}-2 g^{\alpha \beta}\left(U^{v} V_{v}\right) U^{\sigma} \nabla_{[\sigma} V_{\beta]}$.

In relation $(87) K^{\alpha}$ is the relative 4 -acceleration of the particle relatively NRF in IRF coordinates, orthogonal to the 4-velocity $U_{\alpha}, f^{\alpha} /\left(m_{0} c\right)$ is the absolute 4-acceleration of the particle, the last term in (84) contains the reference-frame acceleration and the Coriolis acceleration. Thus, relation (87) is the special relativistic acceleration addition law turning into the classic law in a non-relativistic approximation.

We point out that the relative 4-acceleration appeared as a result of the calculation of the absolute derivative in NRF from the relative 4-velocity of the particle by means of the Christoffel connectivity part (6). Therefore, one can call the curvature tensor (8) $\hat{R}_{\hat{\alpha} \hat{b} \hat{\gamma}}^{\cdots \hat{\mu}}$ as the relative $\mathrm{NRF}$ curvature tensor. Using by the equality

$$
\hat{\nabla}_{\hat{v}} T_{\hat{\mu} \hat{\lambda} .}^{\hat{\gamma}}=\tilde{\nabla}_{\hat{v}} T_{\hat{\mu} \hat{\lambda} .}^{\hat{\gamma}}-T_{\hat{v} \hat{\varepsilon} .}^{\hat{\gamma}} T_{\hat{\mu} \hat{\lambda} .}^{\hat{\varepsilon}}+T_{\hat{v} \hat{\mu} .}^{\hat{\varepsilon}} T_{\hat{\varepsilon} \hat{\lambda} .}^{\hat{\gamma}}+T_{\hat{\nu} \hat{\lambda} .}^{\hat{\varepsilon}} T_{\hat{\mu} \hat{\varepsilon} .}^{\hat{\gamma}},
$$


one can rewrite the curvature tensor (8) in the form

$$
\hat{R}_{\hat{v} \hat{\mu} \hat{\lambda}}^{\cdots \hat{\gamma}}=-2 \tilde{\nabla}_{[\hat{v}} T_{\hat{\mu}] \hat{\lambda} .}^{\hat{\gamma}}-2 T_{[\hat{v}|\hat{\lambda}| \cdot \hat{\mu}] \hat{\varepsilon} .}^{\hat{\varepsilon}} T^{\hat{\gamma}}
$$

Using equality (80) and convolving the curvature tensor (89) by means of Lame coefficients we obtain the expression for the relative curvature tensor in Minkovsky space

$h_{\hat{\gamma}}^{\sigma} h_{\alpha}^{\hat{v}} h_{\beta}^{\hat{\mu}} h_{\delta}^{\hat{\lambda}} \hat{R}_{\hat{\nu} \hat{\mu}, \hat{\lambda}}^{\cdots, \hat{\lambda}}=R_{\alpha \beta, \delta}^{\ldots \sigma}=-2 \partial_{[\alpha} T_{\beta] \delta .}^{\sigma}-2 T_{[\alpha|\delta|}^{\varepsilon} T_{\beta]] \varepsilon}^{\sigma}$.

The $T_{v \mu \text {. }}^{\varepsilon}$ tensor in Minkovsky space has the form

$$
\begin{aligned}
& T_{\nu \mu .}^{\varepsilon}=F^{\varepsilon} V_{v} V_{\mu}-V^{\varepsilon}\left(\Omega_{v \mu}+F_{\mu} V_{v}\right)+ \\
& +V_{v} \Omega_{\mu .}^{\varepsilon}+V_{\mu} \Omega_{v .}^{\varepsilon}
\end{aligned}
$$

After transformations one can represent the tensor in the form

$$
\begin{aligned}
& T_{v \mu, \varepsilon}=\frac{1}{2}\left[F_{v \mu} V_{\varepsilon}+F_{\varepsilon v} V_{\mu}+F_{\varepsilon \mu} V_{v}\right], \\
& F_{\mu \nu}=2 \nabla_{[v} V_{\mu]} .
\end{aligned}
$$

The tensor field of the relative NRF curvature tensor arises in the flat space-time as the result of the division of zero non-holonomic curvature tensor into two nonzero parts. This field is not destroyed by no holonomic transformations both containing the time and not containing the time. We point out that in formula (90) when using the curvilinear coordinates in Minkovsky space the partial derivatives are substituted by the covariant ones.

Although the form of the relative curvature tensor (90) is similar to the Riemann-Christoffel tensor, however the true tensors of the affine connectivity deformation determined in (6) and expressed in the Eulerian coordinates of the Minkovsky space (92) are included in the tensor instead of the connectivity coefficients (not tensors).

For example, we consider the nonrelativistic irrotational dust motion in the Newton gravitational field. We save the terms with the multiplier no more then $1 / c^{2}$ in the relative curvature tensor and in the relative Ricci tensor. In this approximation from (29) and (36) we have

$$
\begin{aligned}
& \tilde{R}_{\hat{a} \hat{b}, \hat{c} \hat{q}}=0, \tilde{R}_{\hat{a} \hat{b}, \hat{c} \hat{0}} \approx 0, \tilde{R}_{\hat{0} \hat{b}, \hat{c} \hat{0}} \approx-\hat{\nabla}_{(\hat{b}} F_{\hat{c})}, \\
& \tilde{R}_{\hat{b} \hat{c}} \approx-\hat{\nabla}_{(\hat{b}} F_{\hat{c})}, \tilde{R}_{\hat{b} \hat{0}} \approx 0, \tilde{R}_{\hat{0} \hat{0}} \approx-\hat{\nabla}_{\hat{n}} F^{\hat{n}} .
\end{aligned}
$$

As $F^{\hat{b}}$ are the space components of the 4-acceleration, then in the non-relativistic case when motion in the Newton field $F^{\hat{b}}=a^{\hat{b}} / c^{2}$, where $a^{\hat{b}}$ is the usual three-dimensional acceleration. From the Poisson equation we obtain

$-\hat{\nabla}_{\hat{n}} a^{\hat{n}}=4 \pi k \rho$,

where $k$ is the gravitational constant, $\varrho$ is the medium density. As a result we have

$$
\tilde{R}_{\hat{0} \hat{0}}=\frac{4 \pi k \rho}{c^{2}}, \tilde{R}_{\hat{b} \hat{0}} \approx 0, \tilde{R}_{\hat{b} \hat{c}}=-\frac{1}{c^{2}} \hat{\nabla}_{(\hat{b}} F_{\hat{c})} .
$$

The first and the second equalities in expression (95) coincide with the corresponding Einstein equations in the synchronous reference frame, the last equality does not coincide.

Let us consider the simplest properties of the relative curvature tensor in Minkovsky space. It follows from expression (92) for the tensor of the affine connectivity deformation $T_{v \mu}^{\varepsilon}$ in Minkovsky space that for the irrotational motions it has the form

$T_{\nu \mu .}^{\varepsilon}=F^{\varepsilon} V_{v} V_{\mu}-V^{\varepsilon} F_{\mu} V_{v}=g^{\varepsilon \sigma} V_{v}\left(\frac{\partial V_{\sigma}}{\partial x^{\mu}}-\frac{\partial V_{\mu}}{\partial x^{\sigma}}\right)$.

If the irrotational motion is rigid, this results in zero relative curvature tensor. Thus, the translation motion of the relativistic rigid body does not result in the appearance of the relative space-time curvature.

For arbitrary motions the relative Ricci tensor can be represented in the from

$$
R^{\beta \gamma}=-\frac{\partial T^{\beta \gamma \alpha}}{\partial x^{\alpha}}-\frac{\partial F^{\gamma}}{\partial x_{\beta}}+\Omega_{\alpha \varepsilon} \Omega^{\alpha \varepsilon} V^{\beta} V^{\gamma}-\Omega^{\gamma \varepsilon} F_{\varepsilon} V^{\beta} .
$$

Scalar relative curvature $R$ is calculated in accordance with the formula 


$$
R=-2 \frac{\partial F^{\gamma}}{\partial x^{\gamma}}+\Omega_{\alpha \varepsilon} \Omega^{\alpha \varepsilon} .
$$

The relative Einstein tensor $G^{\beta \gamma}$ is determined by the expression

$$
\begin{gathered}
G^{\beta \gamma}=-\frac{\partial T^{\beta \gamma \alpha}}{\partial x^{\alpha}}-\frac{\partial F^{\gamma}}{\partial x_{\beta}}+\Omega_{\alpha \varepsilon} \Omega^{\alpha \varepsilon} V^{\beta} V^{\gamma}- \\
-\Omega^{\gamma \varepsilon} F_{\varepsilon} V^{\beta}+g^{\beta \gamma} \frac{\partial F^{\alpha}}{\partial x^{\alpha}}-\frac{1}{2} g^{\beta \gamma} \Omega_{\alpha \varepsilon} \Omega^{\alpha \varepsilon} .
\end{gathered}
$$

For irrotational motions the relative Ricci tensors and the Einstein tensors can be represented in the form

$$
\begin{aligned}
& R^{\beta \gamma}=\frac{\partial\left(F^{\gamma \alpha} V^{\beta}\right)}{\partial x^{\alpha}}-\frac{\partial F^{\gamma}}{\partial x_{\beta}}, \\
& G^{\beta \gamma}=\frac{\partial\left(F^{\gamma \alpha} V^{\beta}\right)}{\partial x^{\alpha}}-\frac{\partial F^{\gamma}}{\partial x_{\beta}}+g^{\beta \gamma} \frac{\partial F^{\alpha}}{\partial x^{\alpha}}= \\
& =2 \frac{\partial}{\partial x^{\alpha}}\left(g^{* \beta[\gamma} F^{\alpha]}\right)
\end{aligned}
$$

where $g^{* \beta \gamma}$ is the projection operator introduced in (40). One can see from (101) that the relative Einstein tensor for irrotational motions identically satisfies to the conservation law

$$
\frac{\partial G^{\beta \gamma}}{\partial x^{\gamma}} \equiv 0
$$

however it is not a symmetrical one.

\section{RELATIVE CONTINUUM CURVATURE TENSOR IN NEWTON MECHANICS}

Let us investigate in the Newton approximation the space-time metric for observers moving together with the medium neglecting by the value $v^{2} / c^{2}$ as compared with the unit. In this approximation metrics (40) reduces to the form

$$
d S^{2}=c^{2} d t^{2}-\delta_{m n} \frac{\partial \Psi^{m}}{\partial y^{\hat{k}}} \frac{\partial \Psi^{n}}{\partial y^{\hat{l}}} \partial y^{\hat{k}} \partial y^{\hat{l}} .
$$

In metric (103) the Cartesian coordinates are selected as the Euler IRF coordinates, where $g_{\mathrm{mn}}$ $=-\delta_{\mathrm{mn}}, t$ is the Newton absolute time. It should be pointed out that metrics (103) in the general case is the Riemannian one with the flat spatial section. At first this result is improbable, however metric (103) permits simple geometrical and physical interpretation.

For example, let us consider the nonrigid rod, the elements of the rod move along its axis with different velocities. A particle moves near the rod and parallel to it with the velocity exceeding the velocities of the rod particles. We agree that the observers at the rod use the IRF watch of Minkovsky space. Let when the particle caught up with the back end of the rod the watch reading is $t_{1}$, and at the over-taking point the watch showed $t_{2}$. The passing time is $\left(t_{2}-t_{1}\right)$. It is clear that one can calculate the relative length of the particle world line when the rod passing in accordance with Pythagoras theorem. The relative length of the particle world line when the rod has infinitesimal dimensions is determined by formula (103). Interval element (103) is obtained from the pseudoEuclidian interval by means of the motion law $x^{n}=\Psi^{n}\left(y^{\hat{k}}, t\right)$, and the differential with respect to $x^{\mathrm{n}}$ is calculated at the fixed $t$ value, that is it is not a complete. Therefore, the squared interval element obtained by the subtraction of the squared spatial element specified in the Lagrange comoving NRF from the squared time element in the general case results in non-Euclidian space-time with the flat spatial section.

Usually when transiting from IRF to NRF one considers the element of the absolute length of the particle world line. The interval element is obtained from the pseudo-Euclidian interval by means of the motion law $x^{n}=\Psi^{n}\left(y^{\hat{k}}, t\right)$, and the differential with respect to $x^{n}$ is complete. Therefore, the squared element (as opposite to (103)) contains the terms depending on the absolute particle velocity, $g_{00}$ component changes and $g_{0 \mathrm{k}}$ components of the metric tensor differed from zero appear. However the space-time remains flat. Obviously, the absolute 
length of the world line of the considered particle is not equal to the relative length of the world line of the particle.

The spatial metric in the Lagrange comoving NRF in accordance with (103) has the form

$$
\hat{\gamma}_{\hat{k} \hat{l}}=\delta_{m n} \frac{\partial \Psi^{m}}{\partial y^{\hat{k}}} \frac{\partial \Psi^{n}}{\partial y^{\hat{l}}} \text {. }
$$

As is known from the continuum mechanics [16]

$$
\frac{d \hat{\gamma}_{\hat{k} \hat{l}}}{d t}=2 \hat{\sigma}_{\hat{k} \hat{l}},
$$

where $\hat{\sigma}_{\hat{k} \hat{l}}$ is the tensor of deformation rates in the comoving RF (reference system). As when moving Lagrange coordinates $y^{\hat{k}}$ of each particle remain constant, then $d y^{\hat{k}} / d t=0$, and therefore

$$
\frac{d \hat{\gamma}_{\hat{k} \hat{l}}}{d t}=\frac{\partial \hat{\gamma}_{\hat{k} \hat{l}}}{d t}+\frac{\partial \hat{\gamma}_{\hat{k} \hat{l}}}{d y^{\hat{m}}} \frac{d \hat{\gamma}^{\hat{m}}}{d t}=\frac{\partial \hat{\gamma}_{\hat{k} \hat{l}}}{d t}=2 \hat{\sigma}_{\hat{k} \hat{l}} .
$$

Let us consider the motion of the airfree gas in the Newton gravitational field using the motion equation in the Euler form and the continuity equation

$$
\frac{\partial v_{a}}{\partial t}+v^{k} \frac{\partial v_{a}}{\partial x^{k}}=g_{a}, \frac{\partial \rho}{\partial t}+\frac{\partial}{\partial x^{a}}\left(\rho v^{a}\right)=0
$$

Differentiating equation (107) with respect to $x^{b}$ we have

$$
\begin{aligned}
& \frac{\partial}{\partial t}\left(\sigma_{a b}+\omega_{a b}\right)+\left(\sigma_{k b}+\omega_{k b}\right)\left(\sigma_{a k}+\omega_{a k}\right)+ \\
& +v^{k} \frac{\partial}{\partial x^{k}}\left(\sigma_{a b}+\omega_{a b}\right)=\frac{\partial g_{a}}{\partial x^{b}}
\end{aligned}
$$

or

$\frac{d}{d t}\left(\sigma_{a b}+\omega_{a b}\right)+\left(\sigma_{k b}+\omega_{k b}\right)\left(\sigma_{a k}+\omega_{a k}\right)=\frac{\partial g_{a}}{\partial x^{b}}$,

where

$$
\sigma_{a b}=\frac{1}{2}\left(\frac{\partial v_{a}}{\partial x^{b}}+\frac{\partial v_{b}}{\partial x^{a}}\right), \omega_{a b}=\frac{1}{2}\left(\frac{\partial v_{a}}{\partial x^{b}}-\frac{\partial v_{b}}{\partial x^{a}}\right) .
$$

In (110) $\sigma_{a b}, \omega_{a b}$ are the deformation and the rotational velocity tensors in the non-relativistic mechanics in the Euler variables.

Convolving (108) on $a, b$ we obtain in the Lagrangian coordinates $\frac{\partial}{\partial t} \hat{\sigma}_{\hat{a}}^{\hat{a}}+\hat{\sigma}_{\hat{b}}^{\hat{k}} \hat{\sigma}_{\hat{k}}^{\hat{b}}=\frac{\partial g_{a}}{\partial x^{a}}, \sigma_{a b}=\frac{\partial v_{a}}{\partial x^{b}}=\frac{\partial v_{b}}{\partial x^{a}}$,

$\frac{\partial \sigma_{a}^{b}}{\partial x^{b}}=\frac{\partial \sigma_{b}^{b}}{\partial x^{a}}$.

The last relation in the Lagrangian coordinates is reduced to the form

$$
\hat{\nabla}_{\hat{b}} \hat{\sigma}_{\hat{a}}^{\hat{b}}-\hat{\nabla}_{\hat{a}} \hat{\sigma}_{\hat{b}}^{\hat{b}}=0 \text {, }
$$

where the covariant derivatives are calculated in accordance with metric (104). To calculate the Ricci tensor we use metrics (103) and the result [10] for the synchronous frame of reference with the flat spatial metric

$$
\begin{aligned}
& \hat{R}_{\hat{0} \hat{0}}=-\frac{1}{c^{2}}\left(\frac{\partial}{\partial t} \hat{\sigma}_{\hat{a}}^{\hat{a}}+\hat{\sigma}_{\hat{b}}^{\hat{k}} \hat{\sigma}_{\hat{k}}^{\hat{b}}\right), \\
& \hat{R}_{\hat{0} \hat{a}}=\frac{1}{c}\left(\hat{\nabla}_{\hat{b}} \hat{\sigma}_{\hat{a}}^{\hat{b}}-\hat{\nabla}_{\hat{a}} \hat{\sigma}_{\hat{b}}^{\hat{b}}\right), \\
& \hat{R}_{\hat{a} \hat{b}}=\frac{1}{c^{2}}\left(\frac{\partial}{\partial t}\left(\hat{\sigma}_{\hat{a} \hat{b}}\right)+\hat{\sigma}_{\hat{a} \hat{b}} \hat{\sigma}_{\hat{k}}^{\hat{k}}-2 \hat{\sigma}_{\hat{a}}^{\hat{k}} \hat{\sigma}_{\hat{b} \hat{k}}\right) .
\end{aligned}
$$

One can show that when the rotations are absent the equality is valid

$$
\frac{\partial}{\partial t} \hat{\sigma}_{\hat{a} \hat{b}}=\left(\frac{d \sigma_{k l}}{d t}+2 \sigma_{m l} \sigma_{m k}\right) \frac{\partial \Psi^{k}}{\partial y^{\hat{a}}} \frac{\partial \Psi^{l}}{\partial y^{\hat{b}}},
$$

using the equality for expression (115) we have

$$
\hat{R}_{\hat{a} \hat{b}}=\frac{1}{c^{2}}\left(\frac{d \sigma_{k l}}{d t}+\sigma_{k l} \sigma_{m}^{m}\right) \frac{\partial \Psi^{k}}{\partial y^{\hat{a}}} \frac{\partial \Psi^{l}}{\partial y^{\hat{b}}} .
$$

Taking into account (109) when the rotations are absent we find

$$
\hat{R}_{\hat{a} \hat{b}}=\frac{1}{c^{2}}\left(\frac{\partial g_{k}}{\partial x^{l}}+\sigma_{k l} \sigma_{m}^{m}-\sigma_{k m} \sigma_{l}^{m}\right) \frac{\partial \Psi^{k}}{\partial y^{\hat{a}}} \frac{\partial \Psi^{l}}{\partial y^{\hat{b}}} .
$$

The medium moving in the eigen gravitational field has $g_{\alpha}=\partial \phi / \partial x^{\alpha}$, where $\phi$ is the potential of the gravitational field satisfying to the Poisson equation. From relations (111) and the Poisson equation for (113) we find

$$
\hat{R}_{\hat{0} \hat{0}}=\frac{4 \pi k \rho}{c^{2}} \text {. }
$$

Relation (114) taking into account (112) forms

$\hat{R}_{\hat{0} \hat{a}}=0$. 
We present expression (118) in aggregate with the Poisson equation in the form convenient for further investigations

$\hat{R}_{\hat{a} \hat{b}}=\frac{4 \pi \rho k}{c^{2}} \hat{\gamma}_{\hat{a} \hat{b}}+\hat{F}_{\hat{a} \hat{b}}$,

$\hat{F}_{\hat{a} \hat{b}}=\left(\frac{\partial g^{m}}{\partial x^{m}} \delta_{k l}+\frac{\partial g_{k}}{\partial x^{l}}+\sigma_{k l} \sigma_{m}^{m}-\sigma_{k m} \sigma_{l}^{m}\right) \frac{\partial \Psi^{k}}{\partial y^{\hat{a}}} \frac{\partial \Psi^{l}}{\partial y^{\hat{b}}}$.

Relations (119)-(121) provided that in the last expression $\hat{F}_{\hat{a} \hat{b}}=0$ are the Einstein equations written in the synchronous frame of reference for the dust-like matter [10]. Obviously, that in the general case $\hat{F}_{\hat{a} \hat{b}} \neq 0$, as at the same force field the congruencies of the world lines of the medium particles have a greater arbitrariness.

Let us ascertain at what particular conditions the NRF geometry determined with the laws of the Newton mechanics and the geometry of the synchronous frame of reference for the dust-like matter determined with the Einstein equations coincide. It follows from metric (104) that the sought for solutions of the Einstein equation in the case of flat spatial sections are valid. And the coincidence of the Einstein equation solutions with the solutions of the Newton mechanics is possible if one imposes the constrain on the congruous of the world lines of the basis particles

$$
\hat{F}_{\hat{a} \hat{b}}=0 \text {. }
$$

Let us investigate the spherical-symmetrical continuum motions with the following velocity field in Euler variables in Cartesian coordinates

$$
v_{a}=v(r, t) n_{a}, n_{a}=\frac{x_{a}}{r}, n_{a} n_{a}=1
$$

Using Euler equations (107) and symmetry conditions (123) we obtain the expression for system (122)

$$
\frac{1}{r} \frac{\partial v}{\partial t}=\Delta \phi, \frac{1}{r} \frac{\partial v}{\partial t}+\frac{v^{2}}{r^{2}}+\frac{2}{r} \frac{\partial \phi}{\partial r}=\Delta \phi .
$$

Let us consider some particular cases of the system solution:

1. For the radial motion of the airfree medium in the Newton central symmetrical gravitational field created by the massive solid with the mass center located at the origin of coordinates we have

$$
\Delta \varphi=0, \varphi=-\frac{k M_{0}}{r}, v^{2}=2 k M_{0}\left(\frac{1}{r}-\frac{1}{r_{0}}\right)+v_{0}^{2},
$$

where $M_{0}$ is the mass of the solid created the field, $\nu_{0}$ is the velocity at $r=r_{0}$. From the compatibility of expressions (124) and (125) we obtain

$$
\frac{\partial v}{\partial t}=0, v^{2}=2 k M_{0} \frac{1}{r} \text {. }
$$

Solution (126) is the particular case of (125) provided the medium at the infinity is at rest. Integrating (126,) we obtain

$$
r= \pm\left(\frac{3 c}{2}\right)^{2 / 3} F^{1 / 3}\left(t_{0}-t\right)^{2 / 3}, F \equiv \frac{2 K M_{0}}{c^{2}}=r_{g}
$$

where $r_{\mathrm{g}}$ is the gravitational radius. We point out that in the latter relation the light velocity $c$ is artificially introduced for the convenience of the comparison with other results, and as a result, it is cancelled in this formula as it should be when integrating the motion equations in the non-relativistic mechanics. Selection of the sign depends on the character of the particle motion. When moving on the radius to the center the sign "plus" is selected, and the sign "minus" is selected when extension from the center. Constant $t_{0}$ is selected from the demand that at $t=0$ it should be $r=r_{0}$, where $r_{0}$ is the Lagrange coordinate. Obviously when falling the particles on the center the current radius of the Lagrange particle $r\left(r_{0}, t\right)$ is decreased, therefore $t<t_{0}$.

The metrics (103) in the spherical coordinate system has the form

$$
\begin{aligned}
& d S^{2}=c^{2} d t^{2}-\left(\frac{\partial r}{\partial r_{0}}\right)^{2} d r_{0}^{2}- \\
& -r^{2}\left(d \theta^{2}+\sin ^{2} \theta d \varphi^{2}\right) .
\end{aligned}
$$

Using the law of motion (127) and considering that

$$
R \equiv \frac{2}{3} \frac{r_{0}^{3 / 2}}{r_{g}^{1 / 2}}
$$


we have the expression for the interval element

$$
\begin{aligned}
& d S^{2}=c^{2} d t^{2}-\frac{d R^{2}}{\left[\frac{3}{2 r_{g}}(R-c t)\right]^{2 / 3}}- \\
& -\left[\frac{3}{2}(R-c t)\right]^{4 / 3} r_{g}^{2 / 3}\left(d \theta^{2}+\sin ^{2} \theta d \varphi^{2}\right),
\end{aligned}
$$

which exactly coincides with the known Lemaitre metric in GR [10]. For our case the interval element of the Lemaitre metrics means the squared relative length of the test particle world line moving relatively free falling on the radius to the center of non-interacting with each other particles in the Newton central symmetrical gravitational field. The falling particles having zero velocity at the infinity form the NRF basis. The character of the forces acting on the test particle does not matter.

Although metric (130) is identical to the corresponding GR metric, however in our case the coordinates and the time determining the metric have a clear metric sense that is absent in GR in principle. For example, the fall time $T$ of the basis particle from the initial radius value $r_{1}$ to the current value $r\left(r_{1}, T\right)$ is the finite value and it is determined by formula

$$
T=\frac{2}{3}\left[\frac{r_{1}}{c}\left(\frac{r_{1}}{r_{g}}\right)^{1 / 2}-\frac{r}{c}\left(\frac{r}{r_{g}}\right)^{1 / 2}\right],
$$

which corresponds to the GR formula when the particle proper time is used as the time [17]. In our case the Newton time $t$ plays the role of the proper time.

2. Following to $[10,17]$ we consider the Newton uniform isotropic cosmological model, for which we have

$v(r, t)=H(t) r$.

Taking into account Euler equations, we write system (124) in the form

$\frac{1}{r} \frac{\partial v}{\partial t}=-4 \pi k \rho, \frac{3}{r} \frac{\partial v}{\partial t}+\frac{v^{2}}{r^{2}}+\frac{2 v}{r} \frac{\partial v}{\partial r}=-4 \pi k \rho$.

From equations (132), (133) we find $\frac{\partial H}{\partial t}=-4 \pi k \rho, \frac{\partial H}{\partial t}+H^{2}=-\frac{4}{3} \pi k \rho$.

Whence

$H^{2}=\frac{8}{3} \pi k \rho$,

that corresponds to the extension case when the density is equal to the critical one. Since the law of the Universe evolution in the Newton approximation was derived in [17] for the arbitrary density, then using the results [17] we find the law of extension for our case

$r=r_{0}\left(\frac{t-t_{\infty}}{t_{0}-t_{\infty}}\right)^{2 / 3}$,

where $\left(t_{0}-t_{\infty}\right)$ is the "age" of the homogeneous model of the Universe. Substitution of (136) into (128) results in the expression for the squared interval

$$
\begin{aligned}
& d S^{2}=c^{2} d t^{2}- \\
& -\left(\frac{t-t_{\infty}}{t_{0}-t_{\infty}}\right)^{4 / 3}\left[d r_{0}^{2}-r_{0}^{2}\left(d \theta^{2}+\sin ^{2} \theta d \varphi^{2}\right)\right],
\end{aligned}
$$

which corresponds to the model with the flat (Euclidean) GR space [18-25].

\section{CONCLUSION}

The strangest result obtained in this work is that the exact solutions of the Einstein equations are contained as the particular cases of the non-relativistic Newton mechanics but not vice-versa.

\section{REFERENCES}

1. Podosenov SA. Geometricheskie svoystva neinertsial'nykh sistem otscheta $v$ relyativistskoy mekhanike. I kn.: Diskussionnye voprocy teorii otnositel'nosti i gravitatsii. [Geometrical properties of noninertial reference frames in relativistic mechanics. In: Debatable problems of theory of relativity and gravitation]. Moscow, Nauka Publ., 1982, p. $95-103$.

2. Podosenov SA. Prostranstvo, vremya $i$ klassicheskie polya syyazannykh struktur [Space, time and classical fields of bound 
structures]. Moscow, Sputnik+ Publ., 2000, $445 \mathrm{p}$.

3. Podosenov SA, Potapov AA, Sokolov AA. Impul'snaya elektrodinamika shirokopolosnykh radiosistem i polya svyazannykh struktur [Pulse electrodynamics of wideband radio systems and fields of bound structures]. Edited by AA. Potapov. Moscow, Radiotekhnika Publ., 2003, 720 p.

4. Podosenov SA. Novy metod rascheta poley v prostranstve-vremeni svyazannykh struktur [New method of field calculation in space-time of bound structures]. Monograph. LAP LAMBERT Academic Publishing, 2011.

5. Podosenov SA, Potapov AA, Foukzon J, Men'kova ER. Negolonomnye, fraktal'nye $i$ svazannye struktury v relyativistskikh sploshnykh sredakh, elektrodinamike, kvantovoy mekhanike i kosmologii.V 3-khtt.Podred.d.f.-m.n.,prof. AA Potapova. T.1: Teoriya impul'snogo izlucheniya $i$ vzaimodeystvie poley s golonomnymi i fraktal'nymi ob'ektami [Nonholonomic, Fractal and Bound Structures in Relativistic Continua, Electrodynamics, Quantum Mechanics and Cosmology. In three books. Book 2. Force Fields in Bound and Nonholonomic Structures]. Moscow, LENAND URSS, 2015, 432 p.

6. Podosenov SA. Struktura tenzora krivizny NSO v STO. V kn.: Teoriya otnositel'nosti $i$ gravitatsiya [Structure of NRF curvature tensor in SRT. In: Theory of relativity and gravitation]. Moscow, Nauka Publ., 1976, p. 107-114.

7. Schouten Jan Arnoldus. Tensor Analysis for Physicists, 2d edn., New York: Dover Publications, 1989.

8. Zel'manov AL. Khronometricheskie invarianty i soputstvuyushchie koordinaty v obshchey teorii otnositel'nosti [Chronometric invariants and comoving coordinates in general relativity theory].
DAN SSSR, 1956, 107(6):815-818 (in Russ.).

9. Podosenov SA. Diss. na soiskanie uch. step. kand. fir:-mat.nauk. Relyativistskaya mekbanika deformiruemoy sredy $v$ tetradnoy formulirovke [Thesis Ph. D. Phys.-math. Relativistic deformable medium mechanics in tetrad formulation]. Moscow, UDN Publ., 1972.

10. Landau LD, Lifshitz EM. The Classical Theory of Fields, 4th ed. Butterworth-Heinemann, 1975, $429 \mathrm{p}$

11. Rashevsky PK. Rimanova geometriya i tenzorny analiz [Riemann geometry and tensor analysis]. Moscow, Nauka Publ., 1967.

12. Dehnen H. Über allgemein-relativistische Dynamik. Wissensch. Zeitschr. der FriedrichSchiller Universitat, Jena, Math.-Naturw. Reihe. H. 1. Jahrg., 1966, 15:15-23.

13. Rodichev VI. Teoriya tyagoteniya vortogonal'nom repere [Gravitational theory in orthogonal frame]. Moscow, Nauka Publ., 1974.

14. Sommerfeld A. Electrodynamik. Vorles. üb. Theor. Phys., Band 3, Geest\&Portig K.G. Verlag, Leipzig, 1949.

15. Podosenov SA. Geometricheskie svoystva soputstvuyushchikh sistem otscheta v spetsial'noy teorii otnositel'nosti. $V$ kn.: Teoriya otnositel'nosti $i$ gevitatsiya [Geometrical properties of comoving reference frames in special theory of relativity. In book: Theory of relativity and gravitation]. Moscow, Nauka Publ., 1976:100-106.

16. Sedov LI. A course in continuum mechanics. Volumes I-IV. Wolters-Noordhoff Publishing, Netherlands, 1971.

17. Zel'dovich YaB, Novikov ID. Relyativistskaya astrofizika [Relativistic astrophysics]. Moscow, Nauka Publ., 1967.

18. Podosenov SA, Potapov AA, Foukzon J. Electrodynamics of a Continuous Medium in a System with Specified Structure. Physics of Wave Phenomena, 2012, 20(2):143-157. 
19. Podosenov SA, Foukzon J, Potapov AA. A Study of the Motion of a Relativistic Continuous Medium. Gravitation and Cosmology, 2010, 16(4):307.

20. Foukzon J, Podosenov SA, Potapov AA. Relativistic length expansion in general accelerated system revisited. http://arxiv. $\mathrm{org} /$ pdf/0910.2298v1.

21. Podosenov SA, Potapov AA, Foukzon J, Men'kova ER. Geometry of Noninertial Bases in Relativistic Mechanics Continua and Bell's Problem Solution. International Journal of Recent Advances in Physics (IJRAP). Wireilla Scientific Publications, 2014, 3(1):23-37. http://wireilla.com/physics/ ijrap/current2014.html.

22. Podosenov SA, Foukzon J, Potapov AA, Men'kova ER. About Nonlinear Classic Field Theory of Connected Charges. International Journal of Recent Advances in Physics (IJRAP). Wireilla Scientific Publications, 2014, 3(2):1-20.

23. Podosenov SA, Foukzon J, Potapov AA, Men'kova ER. About Modelling of the Gravitational Fields. International Journal of Recent Advances in Physics (IJRAP). Wireilla Scientific Publications, 2015, 4(1):1-19.

24. Podosenov SA, Foukzon J, Potapov AA, Men'kova ER. Classical and Quasi-classical Consideration of Charged Particles in Coulomb Field of Bound Charges. International Journal of Recent Advances in Physics (IJRAP). Wireilla Scientific Publications, 2015, 4(1):67-89.

25. Podosenov S.A. Uravneniya struktury $\mathrm{V}$ ralyativistskoy mekhanike sploshnykh sred i reshenie paradoksa Bella [Structure equations in relativistic mechanics of continuum and Bell's paradox solution]. Souremennye naukoemkie tekhnologii. 2014, 3:132-138 (in Russ.). 